\title{
GENERALIZED LIE BIALGEBROIDS AND JACOBI STRUCTURES
}

\author{
David Iglesias, Juan C. Marrero \\ Departamento de Matemática Fundamental, Facultad de Matemáticas, \\ Universidad de la Laguna, La Laguna, \\ Tenerife, Canary Islands, SPAIN, \\ E-mail: diglesia@ull.es, jcmarrer@ull.es
}

\begin{abstract}
The notion of a generalized Lie bialgebroid (a generalization of the notion of a Lie bialgebroid) is introduced in such a way that a Jacobi manifold has associated a canonical generalized Lie bialgebroid. As a kind of converse, we prove that a Jacobi structure can be defined on the base space of a generalized Lie bialgebroid. We also show that it is possible to construct a Lie bialgebroid from a generalized Lie bialgebroid and, as a consequence, we deduce a duality theorem. Finally, some special classes of generalized Lie bialgebroids are considered: triangular generalized Lie bialgebroids and generalized Lie bialgebras.
\end{abstract}

Mathematics Subject Classification (2000): 17B62, 53D10, 53D17.

Key words and phrases: Jacobi manifolds, Poisson manifolds, Lie algebroids, Lie bialgebroids, triangular Lie bialgebroids, Lie bialgebras.

\section{Introduction}

Roughly speaking, a Lie algebroid over a manifold $M$ is a vector bundle $A$ over $M$ such that its space of sections $\Gamma(A)$ admits a Lie algebra structure $\llbracket, \rrbracket$ and, moreover, there exists a bundle map $\rho$ from $A$ to $T M$ which provides a Lie algebra homomorphism from $(\Gamma(A), \llbracket, \rrbracket)$ into the Lie algebra of vector fields $\mathfrak{X}(M)$ (see [22, 25]). Lie algebroids are a natural generalization of tangent bundles and real Lie algebras of finite dimension. But, there are many other interesting examples, for instance, the cotangent bundle $T^{*} M$ of any Poisson manifold $M$ possesses a natural Lie algebroid structure ([1, 2, 4, 28]). In fact, there is a one-to-one correspondence between Lie algebroid structures on a vector bundle $A$ and linear Poisson structures on the dual bundle $A^{*}$ (see [2, [3]). An important class 
of Lie algebroids are the so-called Lie bialgebroids. This is a Lie algebroid $A$ such that the dual vector bundle $A^{*}$ also carries a Lie algebroid structure which is compatible in a certain way with that on $A$ (see [15, 23]). If $M$ is a Poisson manifold, then the pair $\left(T M, T^{*} M\right)$ is a Lie bialgebroid. As a kind of converse, it was proved in [23 that the base space of a Lie bialgebroid is a Poisson manifold. Apart from the pair $\left(T M, T^{*} M\right)$ ( $M$ being a Poisson manifold), other interesting examples of Lie bialgebroids are Lie bialgebras [5]. A Lie bialgebra is a Lie bialgebroid such that the base space is a single point and there is a one-to-one correspondence between Lie bialgebras and connected simply connected Poisson Lie groups (see 15, 21, 28]). We remark that a connected simply connected abelian Poisson Lie group is isomorphic to the dual space of a real Lie algebra endowed with the usual linear Poisson structure (the Lie-Poisson structure). Moreover, Poisson Lie groups are closely related with quantum groups (see [6]).

As it is well-known, a Jacobi structure on a manifold $M$ is a 2-vector $\Lambda$ and a vector field $E$ on $M$ such that $[\Lambda, \Lambda]=2 E \wedge \Lambda$ and $[E, \Lambda]=0$, where [, ] is the Schouten-Nijenhuis bracket [20]. If $(M, \Lambda, E)$ is a Jacobi manifold one can define a bracket of functions, the Jacobi bracket, in such a way that the space $C^{\infty}(M, \mathbb{R})$ endowed with the Jacobi bracket is a local Lie algebra in the sense of Kirillov 14. Conversely, a local Lie algebra structure on $C^{\infty}(M, \mathbb{R})$ induces a Jacobi structure on $M$ [9, 14. Jacobi manifolds are natural generalizations of Poisson manifolds. However, very interesting manifolds like contact and locally conformal symplectic (l.c.s.) manifolds are also Jacobi and they are not Poisson. In fact, a Jacobi manifold admits a generalized foliation whose leaves are contact or l.c.s. manifolds (see [4, 9, 14]). If $M$ is an arbitrary manifold, the vector bundle $T M \times \mathbb{R} \rightarrow M$ possesses a natural Lie algebroid structure. Moreover, if $M$ is a Jacobi manifold then the 1-jet bundle $T^{*} M \times \mathbb{R} \rightarrow M$ admits a Lie algebroid structure [13 (for a Jacobi manifold the vector bundle $T^{*} M$ is not, in general, a Lie algebroid). However, the pair $\left(T M \times \mathbb{R}, T^{*} M \times \mathbb{R}\right)$ is not, in general, a Lie bialgebroid (see [29]).

On the other hand, in 11], we studied Jacobi structures on the dual bundle $A^{*}$ to a vector bundle $A$ such that the Jacobi bracket of linear functions is again linear and the Jacobi bracket of a linear function and the constant function 1 is a basic function. We proved that a Lie algebroid structure on $A$ and a 1-cocycle $\phi_{0} \in \Gamma\left(A^{*}\right)$ induce a Jacobi structure on $A^{*}$ which satisfies the above conditions. Moreover, we showed that this correspondence is a bijection. We also consider two interesting examples: i) for an arbitrary manifold $M$, the Lie algebroid $A=T M \times \mathbb{R}$ and the 1-cocycle $\phi_{0}=(0,1) \in \Omega^{1}(M) \times C^{\infty}(M, \mathbb{R}) \cong \Gamma\left(A^{*}\right)$, we prove that the resultant linear Jacobi structure on $T^{*} M \times \mathbb{R}$ is just the canonical contact structure and ii) for a Jacobi manifold $M$, the Lie algebroid $A^{*}=T^{*} M \times \mathbb{R}$ and the 1cocycle $X_{0}=(-E, 0) \in \mathfrak{X}(M) \times C^{\infty}(M, \mathbb{R}) \cong \Gamma(A)$, we deduce that the corresponding linear Jacobi structure $\left(\Lambda_{\left(T M \times \mathbb{R}, X_{0}\right)}, E_{\left(T M \times \mathbb{R}, X_{0}\right)}\right)$ on $T M \times \mathbb{R}$ is given by

$$
\Lambda_{\left(T M \times \mathbb{R}, X_{0}\right)}=\Lambda^{c}+\frac{\partial}{\partial t} \wedge E^{c}-t\left(\Lambda^{v}+\frac{\partial}{\partial t} \wedge E^{v}\right), \quad E_{\left(T M \times \mathbb{R}, X_{0}\right)}=E^{v},
$$

where $\Lambda^{c}$ (resp. $\Lambda^{v}$ ) is the complete (resp. vertical) lift to $T M$ of $\Lambda$ and $E^{c}$ (resp. $E^{v}$ ) is the complete (resp. vertical) lift to $T M$ of $E$. This Jacobi structure was introduced 
in [10] and it is the Jacobi counterpart to the tangent Poisson structure first used in [26] (see also [3, 8]).

Therefore, for a Jacobi manifold $M$, it seems reasonable to consider the pair $((A=T M \times$ $\left.\left.\mathbb{R}, \phi_{0}=(0,1)\right),\left(A^{*}=T^{*} M \times \mathbb{R}, X_{0}=(-E, 0)\right)\right)$ instead of the pair $\left(T M \times \mathbb{R}, T^{*} M \times \mathbb{R}\right)$. In fact, we prove, in this paper, that the Lie algebroids $T M \times \mathbb{R}$ and $T^{*} M \times \mathbb{R}$ and the 1-cocycles $\phi_{0}$ and $X_{0}$ satisfy some compatibility conditions. These results suggest us to introduce, in a natural way, the definition of a generalized Lie bialgebroid. The aim of this paper is to discuss some relations between generalized Lie bialgebroids and Jacobi structures.

The paper is organized as follows. In Section 2, we recall several definitions and results about Jacobi manifolds and Lie algebroids which will be used in the sequel. In Section 3, we show some facts about the differential calculus on Lie algebroids in the presence of a 1-cocycle. If $(A, \llbracket, \rrbracket, \rho)$ is a Lie algebroid over $M$ and, in addition, we have a 1-cocycle $\phi_{0} \in \Gamma\left(A^{*}\right)$ then the usual representation of the Lie algebra $\Gamma(A)$ on the space $C^{\infty}(M, \mathbb{R})$ can be modified and a new representation is obtained. The resultant cohomology operator $d_{\phi_{0}}$ is called the $\phi_{0^{-}}$-differential of $A$ and its expression, in terms of the differential $d$ of $A$, is $d_{\phi_{0}} \omega=d \omega+\phi_{0} \wedge \omega$, for $\omega \in \Gamma\left(\wedge^{k} A^{*}\right)$. The $\phi_{0}$-differential of $A$ allows us to define, in a natural way, the $\phi_{0}$-Lie derivative by a section $X \in \Gamma(A),\left(\mathcal{L}_{\phi_{0}}\right)_{X}$, as the commutator of $d_{\phi_{0}}$ and the contraction by $X$, that is, $\left(\mathcal{L}_{\phi_{0}}\right)_{X}=d_{\phi_{0}} \circ i_{X}+i_{X} \circ d_{\phi_{0}}$. On the other hand, imitating the definition of the Schouten bracket of two multilinear first-order differential operators on the space of $C^{\infty}$ real-valued functions on a manifold $N$ (see [1]), we introduce the $\phi_{0}$-Schouten bracket of a $k$-section $P$ and a $k^{\prime}$-section $P^{\prime}$ as the $k+k^{\prime}-1$-section given by

$$
\llbracket P, P^{\prime} \rrbracket_{\phi_{0}}=\llbracket P, P^{\prime} \rrbracket+(-1)^{k+1}(k-1) P \wedge\left(i_{\phi_{0}} P^{\prime}\right)-\left(k^{\prime}-1\right)\left(i_{\phi_{0}} P\right) \wedge P^{\prime},
$$

where 【, 』 is the usual Schouten bracket of $A$ (for the properties of the $\phi_{0}$-Schouten bracket, see Theorem 3.5). When $(M, \Lambda, E)$ is a Jacobi manifold and we consider the Lie algebroids $T M \times \mathbb{R}$ and $T^{*} M \times \mathbb{R}$ and the 1-cocycles $\phi_{0}=(0,1)$ and $X_{0}=(-E, 0)$, we prove that the above operators satisfy certain compatibility conditions (see Proposition 3.7). These results suggest us to introduce, in Section 4 , the definition of a generalized Lie bialgebroid as follows. Suppose that $(A, \llbracket, \rrbracket, \rho)$ is a Lie algebroid and that $\phi_{0} \in \Gamma\left(A^{*}\right)$ is a 1-cocycle. Assume also that the dual bundle $A^{*}$ is a Lie algebroid and that $X_{0} \in \Gamma(A)$ is a 1 -cocycle. Then, the pair $\left(\left(A, \phi_{0}\right),\left(A^{*}, X_{0}\right)\right)$ is said to be a generalized Lie bialgebroid over $M$ if for all $X, Y \in \Gamma(A)$ and $P \in \Gamma\left(\wedge^{k} A\right)$

$$
\begin{gathered}
d_{* X_{0}} \llbracket X, Y \rrbracket=\llbracket X, d_{* X_{0}} Y \rrbracket_{\phi_{0}}-\llbracket Y, d_{* X_{0}} X \rrbracket_{\phi_{0}}, \\
\left(\mathcal{L}_{* X_{0}}\right)_{\phi_{0}} P+\llbracket X_{0}, P \rrbracket_{\phi_{0}}=0,
\end{gathered}
$$

where $d_{* X_{0}}$ and $\mathcal{L}_{* X_{0}}$ are the $X_{0}$-differential and the $X_{0}$-Lie derivative, respectively, of $A^{*}$. If the 1-cocycles $\phi_{0}$ and $X_{0}$ are null, we recover the notion of a Lie bialgebroid. Moreover, if $(M, \Lambda, E)$ is a Jacobi manifold, the pair $\left((T M \times \mathbb{R},(0,1)),\left(T^{*} M \times \mathbb{R},(-E, 0)\right)\right)$ is a generalized Lie bialgebroid. In fact, extending a result of 23 for Lie bialgebroids, we 
prove that the base space of a generalized Lie bialgebroid is a Jacobi manifold (Theorem 4.6). It is well-known that the product of a Jacobi manifold with $\mathbb{R}$, endowed with the Poissonization of the Jacobi structure, is a Poisson manifold (see [20] and Section 2.1). We show a similar result for generalized Lie bialgebroids. Namely, we prove that if $\left(\left(A, \phi_{0}\right),\left(A^{*}, X_{0}\right)\right)$ is a generalized Lie bialgebroid over $M$ then it is possible to define a Lie bialgebroid structure on the dual pair of vector bundles $\left(A \times \mathbb{R}, A^{*} \times \mathbb{R}\right)$ over $M \times \mathbb{R}$, in such a way that the induced Poisson structure on $M \times \mathbb{R}$ is just the Poissonization of the Jacobi structure on $M$ (Theorem 4.11). Using this result, we deduce that the generalized Lie bialgebroids satisfy a duality theorem, that is, if $\left(\left(A, \phi_{0}\right),\left(A^{*}, X_{0}\right)\right)$ is a generalized Lie bialgebroid, so is $\left(\left(A^{*}, X_{0}\right),\left(A, \phi_{0}\right)\right)$.

In Section 5, we prove that it is possible to obtain a generalized Lie bialgebroid from a Lie algebroid $(A, \llbracket, \rrbracket, \rho)$, a 1-cocycle $\phi_{0}$ on it and a bisection $P \in \Gamma\left(\wedge^{2} A\right)$ satisfying $\llbracket P, P \rrbracket_{\phi_{0}}=$ 0 (Theorem 5.1). This type of generalized Lie bialgebroids are called triangular. Examples of triangular generalized Lie bialgebroids are the triangular Lie bialgebroids in the sense of [23] and the generalized Lie bialgebroid associated with a Jacobi structure. Finally, in Section 6, we study generalized Lie bialgebras, i.e., generalized Lie bialgebroids over a single point. Using the results of Section 5, we deduce that generalized Lie bialgebras can be obtained from algebraic Jacobi structures on a Lie algebra. This fact allows us to give examples of Lie groups whose Lie algebras are generalized Lie bialgebras. The study of this type of Lie groups is the subject of a forthcoming paper [12].

\section{Jacobi manifolds and Lie algebroids}

Let $M$ be a differentiable manifold of dimension $n$. We will denote by $C^{\infty}(M, \mathbb{R})$ the algebra of $C^{\infty}$ real-valued functions on $M$, by $\Omega^{k}(M)$ the space of $k$-forms, by $\mathcal{V}^{k}(M)$ the space of $k$-vectors, with $k \geq 2$, by $\mathfrak{X}(M)$ the Lie algebra of vector fields, by $\delta$ the usual differential on $\Omega^{*}(M)=\oplus_{k} \Omega^{k}(M)$ and by [, ] the Schouten-Nijenhuis bracket (匹1, 28]).

\section{$2.1 \quad$ Jacobi manifolds}

A Jacobi structure on $M$ is a pair $(\Lambda, E)$, where $\Lambda$ is a 2-vector and $E$ is a vector field on $M$ satisfying the following properties:

$$
[\Lambda, \Lambda]=2 E \wedge \Lambda, \quad[E, \Lambda]=0
$$

The manifold $M$ endowed with a Jacobi structure is called a Jacobi manifold. A bracket of functions (the Jacobi bracket) is defined by

$$
\{f, g\}=\Lambda(\delta f, \delta g)+f E(g)-g E(f),
$$


for all $f, g \in C^{\infty}(M, \mathbb{R})$. In fact, the space $C^{\infty}(M, \mathbb{R})$ endowed with the Jacobi bracket is a local Lie algebra in the sense of Kirillov (see [14]), that is, the mapping $\{\}:, C^{\infty}(M, \mathbb{R}) \times$ $C^{\infty}(M, \mathbb{R}) \rightarrow C^{\infty}(M, \mathbb{R})$ is $\mathbb{R}$-bilinear, skew-symmetric, satisfies the Jacobi identity and

$$
\text { support }\{f, g\} \subseteq \text { support } f \cap \text { support } g \text {, for } f, g \in C^{\infty}(M, \mathbb{R}) \text {, }
$$

or, equivalently, $\{$,$\} is \mathbb{R}$-bilinear, skew-symmetric, satisfies the Jacobi identity and is a first-order differential operator on each of its arguments, with respect to the ordinary multiplication of functions, i.e.,

$$
\left\{f_{1} f_{2}, g\right\}=f_{1}\left\{f_{2}, g\right\}+f_{2}\left\{f_{1}, g\right\}-f_{1} f_{2}\{1, g\} \text {, for } f_{1}, f_{2}, g \in C^{\infty}(M, \mathbb{R}) \text {. }
$$

Conversely, a structure of local Lie algebra on $C^{\infty}(M, \mathbb{R})$ defines a Jacobi structure on $M$ (see [9, 14]). If the vector field $E$ identically vanishes then $(M, \Lambda)$ is a Poisson manifold. Jacobi and Poisson manifolds were introduced by Lichnerowicz (匹19, 20]) (see also [1, 4, [18, 28, 30]).

Remark 2.1 Let $(\Lambda, E)$ be a Jacobi structure on a manifold $M$ and consider on the product manifold $M \times \mathbb{R}$ the 2 -vector $\tilde{\Lambda}$ given by

$$
\tilde{\Lambda}=e^{-t}\left(\Lambda+\frac{\partial}{\partial t} \wedge E\right)
$$

where $t$ is the usual coordinate on $\mathbb{R}$. Then, $\tilde{\Lambda}$ defines a Poisson structure on $M \times \mathbb{R}$ (see [20]). The manifold $M \times \mathbb{R}$ endowed with the structure $\tilde{\Lambda}$ is called the Poissonization of the Jacobi manifold $(M, \Lambda, E)$.

\subsection{Lie algebroids}

A Lie algebroid $A$ over a manifold $M$ is a vector bundle $A$ over $M$ together with a Lie algebra structure on the space $\Gamma(A)$ of the global cross sections of $A \rightarrow M$ and a bundle map $\rho: A \rightarrow T M$, called the anchor map, such that if we also denote by $\rho: \Gamma(A) \rightarrow \mathfrak{X}(M)$ the homomorphism of $C^{\infty}(M, \mathbb{R})$-modules induced by the anchor map then:

(i) $\rho:(\Gamma(A), \llbracket, \rrbracket) \rightarrow(\mathfrak{X}(M),[]$,$) is a Lie algebra homomorphism and$

(ii) for all $f \in C^{\infty}(M, \mathbb{R})$ and for all $X, Y \in \Gamma(A)$, one has

$$
\llbracket X, f Y \rrbracket=f \llbracket X, Y \rrbracket+(\rho(X)(f)) Y .
$$

The triple $(A, \llbracket, \rrbracket, \rho)$ is called a Lie algebroid over $M$ (see [22, 25]).

A real Lie algebra of finite dimension is a Lie algebroid over a point. Another trivial example of a Lie algebroid is the triple $(T M,[],, I d)$, where $M$ is a differentiable manifold and $I d: T M \rightarrow T M$ is the identity map. 
If $A$ is a Lie algebroid, the Lie bracket on the sections of $A$ can be extended to the socalled Schouten bracket $\llbracket, \rrbracket$ on the space $\Gamma\left(\wedge^{*} A\right)=\oplus_{k} \Gamma\left(\wedge^{k} A\right)$ of multi-sections of $A$. The Schouten bracket $\llbracket, \rrbracket: \Gamma\left(\wedge^{k} A\right) \times \Gamma\left(\wedge^{k^{\prime}} A\right) \rightarrow \Gamma\left(\wedge^{k+k^{\prime}-1} A\right)$ is characterized by the following conditions: $\llbracket, \rrbracket: \Gamma(A) \times \Gamma(A) \rightarrow \Gamma(A)$ coincides with the Lie algebroid bracket, $\llbracket X, f \rrbracket=\rho(X)(f)$ for $X \in \Gamma(A)$ and $f \in C^{\infty}(M, \mathbb{R})$ and the properties

$$
\begin{gathered}
\llbracket P, P^{\prime} \rrbracket=(-1)^{k k^{\prime}} \llbracket P^{\prime}, P \rrbracket, \\
\llbracket P, P^{\prime} \wedge P^{\prime \prime} \rrbracket=\llbracket P, P^{\prime} \rrbracket \wedge P^{\prime \prime}+(-1)^{k^{\prime}(k+1)} P^{\prime} \wedge \llbracket P, P^{\prime \prime} \rrbracket, \\
(-1)^{k k^{\prime \prime}} \llbracket \llbracket P, P^{\prime} \rrbracket, P^{\prime \prime} \rrbracket+(-1)^{k^{\prime} k^{\prime \prime}} \llbracket \llbracket P^{\prime \prime}, P \rrbracket, P^{\prime} \rrbracket+(-1)^{k k^{\prime}} \llbracket \llbracket P^{\prime}, P^{\prime \prime} \rrbracket, P \rrbracket=0,
\end{gathered}
$$

holds for all $P \in \Gamma\left(\wedge^{k} A\right), P^{\prime} \in \Gamma\left(\wedge^{k^{\prime}} A\right)$ and $P^{\prime \prime} \in \Gamma\left(\wedge^{k^{\prime \prime}} A\right)$ (see [23]).

Next, we will recall the definition of the Lie algebroid cohomology complex with trivial coefficients. For this purpose, we recall the definition of the cohomology of a Lie algebra $\mathcal{A}$ with coefficients in an $\mathcal{A}$-module (we will follow [28]).

Let $(\mathcal{A},[]$,$) be a real Lie algebra (not necessarily finite dimensional) and \mathcal{M}$ a real vector space endowed with a $\mathbb{R}$-bilinear multiplication

$$
\mathcal{A} \times \mathcal{M} \rightarrow \mathcal{M}, \quad(a, m) \mapsto a \cdot m,
$$

such that

$$
\left[a_{1}, a_{2}\right] \cdot m=a_{1} \cdot\left(a_{2} \cdot m\right)-a_{2} \cdot\left(a_{1} \cdot m\right),
$$

for $a_{1}, a_{2} \in \mathcal{A}$ and $m \in \mathcal{M}$. In other words, we have a representation of $\mathcal{A}$ on $\mathcal{M}$. In such a case, a $k$-linear skew-symmetric mapping $c^{k}: \mathcal{A}^{k} \rightarrow \mathcal{M}$ is called an $\mathcal{M}$-valued $k$-cochain. These cochains form a real vector space $C^{k}(\mathcal{A} ; \mathcal{M})$ and the linear operator $\partial^{k}: C^{k}(\mathcal{A} ; \mathcal{M}) \rightarrow C^{k+1}(\mathcal{A} ; \mathcal{M})$ given by

$$
\begin{aligned}
\left(\partial^{k} c^{k}\right)\left(a_{0}, \ldots, a_{k}\right)= & \sum_{i=0}^{k}(-1)^{i} a_{i} \cdot c^{k}\left(a_{0}, \ldots, \hat{a}_{i}, \ldots, a_{k}\right)+ \\
& \sum_{i<j}(-1)^{i+j} c^{k}\left(\left[a_{i}, a_{j}\right], a_{0}, \ldots, \hat{a}_{i}, \ldots, \hat{a}_{j}, \ldots, a_{k}\right)
\end{aligned}
$$

defines a coboundary since $\partial^{k+1} \circ \partial^{k}=0$. Hence we have the corresponding cohomology spaces

$$
H^{k}(\mathcal{A} ; \mathcal{M})=\frac{\operatorname{ker}\left\{\partial^{k}: C^{k}(\mathcal{A} ; \mathcal{M}) \rightarrow C^{k+1}(\mathcal{A} ; \mathcal{M})\right\}}{\operatorname{Im}\left\{\partial^{k-1}: C^{k-1}(\mathcal{A} ; \mathcal{M}) \rightarrow C^{k}(\mathcal{A} ; \mathcal{M})\right\}} .
$$

This cohomology is called the cohomology of the Lie algebra $\mathcal{A}$ with coefficients in $\mathcal{M}$, or relative to the given representation of $\mathcal{A}$ on $\mathcal{M}$.

Now, if $(A, \llbracket, \rrbracket, \rho)$ is a Lie algebroid, we can define the representation of the Lie algebra $(\Gamma(A), \llbracket, \rrbracket)$ on the space $C^{\infty}(M, \mathbb{R})$ given by $X \cdot f=\rho(X)(f)$, for $X \in \Gamma(A)$ and $f \in C^{\infty}(M, \mathbb{R})$. We will denote by $d$ the cohomology operator of the corresponding cohomology complex. Note that the space of the $k$-cochains which are $C^{\infty}(M, \mathbb{R})$-linear is just $\Gamma\left(\wedge^{k} A^{*}\right)$, where $A^{*}$ is the dual bundle to $A$. Moreover, we have that $d\left(\Gamma\left(\wedge^{k} A^{*}\right)\right) \subseteq$ 
$\Gamma\left(\wedge^{k+1} A^{*}\right)$, for all $k$, and thus one can consider the subcomplex $\left(\Gamma\left(\wedge^{*} A^{*}\right), d_{\mid \Gamma\left(\wedge^{*} A^{*}\right)}\right)$. The cohomology of this subcomplex is the Lie algebroid cohomology with trivial coefficients and the restriction of $d$ to $\Gamma\left(\wedge^{*} A^{*}\right)$ is the differential of the Lie algebroid $A$ (see [22]).

Using the above definitions, it follows that a 1-cochain $\phi \in \Gamma\left(A^{*}\right)$ is a 1-cocycle if and only if

$$
\phi \llbracket X, Y \rrbracket=\rho(X)(\phi(Y))-\rho(Y)(\phi(X)), \text { for all } X, Y \in \Gamma(A) .
$$

To end this section, we will consider two examples of Lie algebroids.

1.- The Lie algebroid $(T M \times \mathbb{R},[],, \pi)$

If $M$ is a differentiable manifold, we will exhibit a natural Lie algebroid structure on the vector bundle $T M \times \mathbb{R}$. First, we will show some identifications which will be useful in the sequel.

Let $A \rightarrow M$ be a vector bundle over $M$. Then, it is clear that $A \times \mathbb{R}$ is the total space of a vector bundle over $M$. Moreover, the dual bundle to $A \times \mathbb{R}$ is $A^{*} \times \mathbb{R}$ and the spaces $\Gamma\left(\wedge^{r}(A \times \mathbb{R})\right)$ and $\Gamma\left(\wedge^{k}\left(A^{*} \times \mathbb{R}\right)\right)$ can be identified with $\Gamma\left(\wedge^{r} A\right) \oplus \Gamma\left(\wedge^{r-1} A\right)$ and $\Gamma\left(\wedge^{k} A^{*}\right) \oplus \Gamma\left(\wedge^{k-1} A^{*}\right)$ in such a way that

$$
\begin{aligned}
& (P, Q)\left(\left(\alpha_{1}, f_{1}\right), \ldots,\left(\alpha_{r}, f_{r}\right)\right)=P\left(\alpha_{1}, \ldots, \alpha_{r}\right)+\sum_{i=1}^{r}(-1)^{i+1} f_{i} Q\left(\alpha_{1}, \ldots, \hat{\alpha}_{i}, \ldots, \alpha_{r}\right), \\
& (\alpha, \beta)\left(\left(X_{1}, g_{1}\right), \ldots,\left(X_{k}, g_{k}\right)\right)=\alpha\left(X_{1}, \ldots, X_{k}\right)+\sum_{i=1}^{k}(-1)^{i+1} g_{i} \beta\left(X_{1}, \ldots, \hat{X}_{i}, \ldots, X_{k}\right),
\end{aligned}
$$

for $(P, Q) \in \Gamma\left(\wedge^{r} A\right) \oplus \Gamma\left(\wedge^{r-1} A\right),(\alpha, \beta) \in \Gamma\left(\wedge^{k} A^{*}\right) \oplus \Gamma\left(\wedge^{k-1} A^{*}\right),\left(\alpha_{i}, f_{i}\right) \in \Gamma\left(A^{*}\right) \oplus$ $C^{\infty}(M, \mathbb{R})$ and $\left(X_{j}, g_{j}\right) \in \Gamma(A) \oplus C^{\infty}(M, \mathbb{R})$, with $i \in\{1, \cdots, r\}$ and $j \in\{1, \cdots, k\}$.

Under these identifications, the contractions and the exterior products are given by

$$
\begin{array}{lll}
i_{(\alpha, \beta)}(P, Q) & =\left(i_{\alpha} P+i_{\beta} Q,(-1)^{k} i_{\alpha} Q\right), & \text { if } k \leq r, \\
i_{(\alpha, \beta)}(P, Q) & =0, & \text { if } k>r, \\
i_{(P, Q)}(\alpha, \beta) & =\left(i_{P} \alpha+i_{Q} \beta,(-1)^{r} i_{P} \beta\right), & \text { if } r \leq k, \\
i_{(P, Q)}(\alpha, \beta) & =0, & \text { if } r>k, \\
(P, Q) \wedge\left(P^{\prime}, Q^{\prime}\right) & =\left(P \wedge P^{\prime}, Q \wedge P^{\prime}+(-1)^{r} P \wedge Q^{\prime}\right), & \\
(\alpha, \beta) \wedge\left(\alpha^{\prime}, \beta^{\prime}\right) & =\left(\alpha \wedge \alpha^{\prime}, \beta \wedge \alpha^{\prime}+(-1)^{k} \alpha \wedge \beta^{\prime}\right), &
\end{array}
$$

for $\left(P^{\prime}, Q^{\prime}\right) \in \Gamma\left(\wedge^{r^{\prime}} A\right) \oplus \Gamma\left(\wedge^{r^{\prime}-1} A\right)$ and $\left(\alpha^{\prime}, \beta^{\prime}\right) \in \Gamma\left(\wedge^{k^{\prime}} A^{*}\right) \oplus \Gamma\left(\wedge^{k^{\prime}-1} A^{*}\right)$.

Now, suppose that $A$ is the tangent bundle $T M$. Then, the triple $(A \times \mathbb{R}=T M \times$ $\mathbb{R},[],, \pi)$ is a Lie algebroid over $M$, where $\pi: T M \times \mathbb{R} \rightarrow T M$ is the canonical projection over the first factor and $[$,$] is the bracket given by (see [24, 29])$

$$
[(X, f),(Y, g)]=([X, Y], X(g)-Y(f)),
$$

for $(X, f),(Y, g) \in \mathfrak{X}(M) \times C^{\infty}(M, \mathbb{R}) \cong \Gamma(T M \times \mathbb{R})$. In this case, the dual bundle to $T M \times \mathbb{R}$ is $T^{*} M \times \mathbb{R}$ and the spaces $\Gamma\left(\wedge^{r}(T M \times \mathbb{R})\right)$ and $\Gamma\left(\wedge^{k}\left(T^{*} M \times \mathbb{R}\right)\right)$ can be 
identified with $\mathcal{V}^{r}(M) \oplus \mathcal{V}^{r-1}(M)$ and $\Omega^{k}(M) \oplus \Omega^{k-1}(M)$. Under these identifications, the differential $\tilde{\delta}$ of the Lie algebroid is

$$
\tilde{\delta}(\alpha, \beta)=(\delta \alpha,-\delta \beta)
$$

and the Schouten bracket [, ] is given by

$$
\left[(P, Q),\left(P^{\prime}, Q^{\prime}\right)\right]=\left(\left[P, P^{\prime}\right],(-1)^{k+1}\left[P, Q^{\prime}\right]-\left[Q, P^{\prime}\right]\right) .
$$

2.- The Lie algebroid $\left(T^{*} M \times \mathbb{R}, \llbracket, \rrbracket_{(\Lambda, E)}, \widetilde{\#}_{(\Lambda, E)}\right)$ associated with a Jacobi manifold $(M, \Lambda, E)$ If $A \rightarrow M$ is a vector bundle over $M$ and $P \in \Gamma\left(\wedge^{2} A\right)$ is a 2-section of $A$, we will denote by $\#_{P}: \Gamma\left(A^{*}\right) \rightarrow \Gamma(A)$ the homomorphism of $C^{\infty}(M, \mathbb{R})$-modules given by

$$
\beta\left(\#_{P}(\alpha)\right)=P(\alpha, \beta) \text {, for } \alpha, \beta \in \Gamma\left(A^{*}\right) .
$$

We will also denote by $\#_{P}: A^{*} \rightarrow A$ the corresponding bundle map.

Then, a Jacobi manifold $(M, \Lambda, E)$ has an associated Lie algebroid $\left(T^{*} M \times \mathbb{R}, \llbracket, \rrbracket_{(\Lambda, E)}\right.$, $\left.\widetilde{\#}_{(\Lambda, E)}\right)$, where $\llbracket, \rrbracket_{(\Lambda, E)}, \widetilde{\#}_{(\Lambda, E)}$ are defined by

$$
\begin{aligned}
\llbracket(\alpha, f),(\beta, g) \rrbracket_{(\Lambda, E)}= & \left(\mathcal{L}_{\#_{\Lambda}(\alpha)} \beta-\mathcal{L}_{\#_{\Lambda}(\beta)} \alpha-\delta(\Lambda(\alpha, \beta))+f \mathcal{L}_{E} \beta-g \mathcal{L}_{E} \alpha-i_{E}(\alpha \wedge \beta),\right. \\
& \left.\Lambda(\beta, \alpha)+\#_{\Lambda}(\alpha)(g)-\#_{\Lambda}(\beta)(f)+f E(g)-g E(f)\right), \\
\widetilde{\#}_{(\Lambda, E)}(\alpha, f)= & \#_{\Lambda}(\alpha)+f E,
\end{aligned}
$$

for $(\alpha, f),(\beta, g) \in \Omega^{1}(M) \times C^{\infty}(M, \mathbb{R}), \mathcal{L}$ being the Lie derivative operator (see [13]). For this algebroid, the differential $d_{*}$ is given by (see [16, 17])

$$
d_{*}(P, Q)=(-[\Lambda, P]+k E \wedge P+\Lambda \wedge Q,[\Lambda, Q]-(k-1) E \wedge Q+[E, P]),
$$

for $(P, Q) \in \mathcal{V}^{k}(M) \oplus \mathcal{V}^{k-1}(M)$.

In the particular case when $(M, \Lambda)$ is a Poisson manifold we recover, by projection, the Lie algebroid $\left(T^{*} M, \llbracket, \rrbracket_{\Lambda}, \#_{\Lambda}\right)$, where $\llbracket, \rrbracket_{\Lambda}$ is the bracket of 1 -forms defined by (see [1, 2, 7, 28]):

$$
\llbracket, \rrbracket_{\Lambda}: \Omega^{1}(M) \times \Omega^{1}(M) \rightarrow \Omega^{1}(M), \quad \llbracket \alpha, \beta \rrbracket_{\Lambda}=\mathcal{L}_{\#_{\Lambda}(\alpha)} \beta-\mathcal{L}_{\#_{\Lambda}(\beta)} \alpha-\delta(\Lambda(\alpha, \beta)) .
$$

For this algebroid, the differential is the operator $d_{*}=-[\Lambda, \cdot]$. This operator was introduced by Lichnerowicz in [19] to define the Poisson cohomology.

\section{Differential calculus on Lie algebroids in the pre- sence of a 1-cocycle}




\section{$3.1 \phi_{0}$-differential and $\phi_{0}$-Lie derivative}

Let $(A, \llbracket, \rrbracket, \rho)$ be a Lie algebroid over $M$ and $\phi_{0} \in \Gamma\left(A^{*}\right)$ be a 1-cocycle in the Lie algebroid cohomology complex with trivial coefficients. Using (2.2), we can define a representation $\rho_{\phi_{0}}: \Gamma(A) \times C^{\infty}(M, \mathbb{R}) \rightarrow C^{\infty}(M, \mathbb{R})$ of the Lie algebra $(\Gamma(A), \llbracket, \rrbracket)$ on the space $C^{\infty}(M, \mathbb{R})$ given by

$$
\rho_{\phi_{0}}(X) f=\rho(X)(f)+\phi_{0}(X) f,
$$

for $X \in \Gamma(A)$ and $f \in C^{\infty}(M, \mathbb{R})$. Thus, one can consider the cohomology of the Lie algebra $(\Gamma(A), \llbracket \mathbb{\rrbracket}$,$) with coefficients in C^{\infty}(M, \mathbb{R})$ and the subcomplex $\Gamma\left(\wedge^{*} A^{*}\right)$ consisting of the cochains which are $C^{\infty}(M, \mathbb{R})$-linear. The cohomology operator $d_{\phi_{0}}: \Gamma\left(\wedge^{k} A^{*}\right) \rightarrow$ $\Gamma\left(\wedge^{k+1} A^{*}\right)$ of this subcomplex is called the $\phi_{0^{-}}$differential of $A$. We have that

$$
d_{\phi_{0}} \omega=d \omega+\phi_{0} \wedge \omega
$$

where $d$ is the differential of the Lie algebroid $(A, \llbracket, \rrbracket, \rho)$. As a consequence,

$$
\begin{gathered}
d_{\phi_{0}} 1=\phi_{0}, \\
d_{\phi_{0}}\left(\omega \wedge \omega^{\prime}\right)=\left(d_{\phi_{0}} \omega\right) \wedge \omega^{\prime}+(-1)^{k} \omega \wedge\left(d_{\phi_{0}} \omega^{\prime}\right)-\phi_{0} \wedge \omega \wedge \omega^{\prime},
\end{gathered}
$$

for $\omega \in \Gamma\left(\wedge^{k} A^{*}\right)$ and $\omega^{\prime} \in \Gamma\left(\wedge^{k^{\prime}} A^{*}\right)$.

Remark 3.1 If $\phi_{0}$ is a closed 1-form on a manifold $M$ then $\phi_{0}$ is a 1-cocycle for the trivial Lie algebroid $(T M,[],, I d)$ and we can consider the operator $d_{\phi_{0}}$. Some results about the cohomology defined by $d_{\phi_{0}}$ were obtained in [9, 16, 27]. These results were used in the study of locally conformal Kähler and locally conformal symplectic structures.

On the other hand, if $k \geq 0$ and $X \in \Gamma(A)$, the $\phi_{0}$-Lie derivative with respect to $X$, $\left(\mathcal{L}_{\phi_{0}}\right)_{X}: \Gamma\left(\wedge^{k} A^{*}\right) \rightarrow \Gamma\left(\wedge^{k} A^{*}\right)$, is defined by

$$
\left(\left(\left(\mathcal{L}_{\phi_{0}}\right)_{X}\right) \omega\right)\left(X_{1}, \ldots, X_{k}\right)=\rho_{\phi_{0}}(X)\left(\omega\left(X_{1}, \ldots, X_{k}\right)\right)-\sum_{i=1}^{k} \omega\left(X_{1}, \ldots, \llbracket X, X_{i} \rrbracket, \ldots, X_{k}\right),
$$

for $\omega \in \Gamma\left(\wedge^{k} A^{*}\right)$ and $X_{1}, \ldots, X_{k} \in \Gamma(A)$. It follows that

$$
\left(\mathcal{L}_{\phi_{0}}\right)_{X} \omega=\mathcal{L}_{X} \omega+\phi_{0}(X) \omega,
$$

$\mathcal{L}$ being the Lie derivative of the Lie algebroid $(A, \llbracket, \rrbracket, \rho)$. Thus,

$$
\left(\mathcal{L}_{\phi_{0}}\right)_{X}=d_{\phi_{0}} \circ i_{X}+i_{X} \circ d_{\phi_{0}}
$$

where $i_{X}$ is the usual contraction by $X$. Using (3.6) and the properties of $\mathcal{L}$ (see [23]), we deduce that 
Proposition 3.2 Let $(A, \llbracket, \rrbracket, \rho)$ be a Lie algebroid over $M$ and $\phi_{0} \in \Gamma\left(A^{*}\right)$ be a 1-cocycle. If $X \in \Gamma(A), f \in C^{\infty}(M, \mathbb{R}), \omega \in \Gamma\left(\wedge^{k} A^{*}\right)$ and $\omega^{\prime} \in \Gamma\left(\wedge^{k^{\prime}} A^{*}\right)$, we have

$$
\begin{gathered}
\left(\mathcal{L}_{\phi_{0}}\right)_{X}\left(\omega \wedge \omega^{\prime}\right)=\left(\left(\mathcal{L}_{\phi_{0}}\right)_{X} \omega\right) \wedge \omega^{\prime}+\omega \wedge\left(\left(\mathcal{L}_{\phi_{0}}\right)_{X} \omega^{\prime}\right)-\phi_{0}(X) \omega \wedge \omega^{\prime}, \\
\left(\mathcal{L}_{\phi_{0}}\right)_{f X} \omega=f\left(\mathcal{L}_{\phi_{0}}\right)_{X} \omega+d f \wedge i_{X} \omega .
\end{gathered}
$$

Now, we will consider the examples of Lie algebroids studied in Section 2.2.

1.- The Lie algebroid $(T M \times \mathbb{R},[],, \pi)$

Using (2.6), it follows that $\phi_{0}=(0,1) \in \Omega^{1}(M) \times C^{\infty}(M, \mathbb{R}) \cong \Gamma\left(T^{*} M \times \mathbb{R}\right)$ is a 1cocycle. Thus, we have the corresponding representation $\pi_{(0,1)}:\left(\mathfrak{X}(M) \times C^{\infty}(M, \mathbb{R})\right) \times$ $C^{\infty}(M, \mathbb{R}) \rightarrow C^{\infty}(M, \mathbb{R})$ of the Lie algebra $\left(\mathfrak{X}(M) \times C^{\infty}(M, \mathbb{R}),[],\right)$ on the space $C^{\infty}(M, \mathbb{R})$ which, in this case, is given by (see (3.1))

$$
\pi_{(0,1)}((X, f), g)=X(g)+f g,
$$

for $(X, f) \in \mathfrak{X}(M) \times C^{\infty}(M, \mathbb{R})$ and $g \in C^{\infty}(M, \mathbb{R})$. From (2.3), (2.6) and (3.2), we deduce that the $\phi_{0}$-differential $\tilde{\delta}_{\phi_{0}}=\tilde{\delta}_{(0,1)}$ is given by

$$
\tilde{\delta}_{(0,1)}(\alpha, \beta)=(\delta \alpha, \alpha-\delta \beta),
$$

for $(\alpha, \beta) \in \Omega^{k}(M) \oplus \Omega^{k-1}(M)$.

Remark 3.3 i) If $(\Lambda, E) \in \Gamma\left(\wedge^{2}(T M \times \mathbb{R})\right)$ is a Jacobi structure on $M$ a long computation, using (2.3), (2.4), (3.7) and (3.11), shows that the Lie algebroid bracket $\llbracket, \rrbracket_{(\Lambda, E)}$ and the anchor map $\tilde{\#}_{(\Lambda, E)}$ can be written using the homomorphism $\#_{(\Lambda, E)}: \Gamma\left(T^{*} M \times \mathbb{R}\right) \rightarrow$ $\Gamma(T M \times \mathbb{R})$ and the operators $\mathcal{L}_{(0,1)}$ and $\tilde{\delta}_{(0,1)}$ as follows

$$
\begin{aligned}
\llbracket(\alpha, f),(\beta, g) \rrbracket_{(\Lambda, E)}= & \left(\mathcal{L}_{(0,1)}\right)_{\#(\Lambda, E)}(\alpha, f)(\beta, g)-\left(\mathcal{L}_{(0,1)}\right)_{\#(\Lambda, E)}(\beta, g) \\
& -\tilde{\delta}_{(0,1)}((\Lambda, E)((\alpha, f),(\beta, g))) \\
= & i_{\#_{(\Lambda, E)}(\alpha, f)}\left(\tilde{\delta}_{(0,1)}(\beta, g)\right)-i_{\#(\Lambda, E)(\beta, g)}\left(\tilde{\delta}_{(0,1)}(\alpha, f)\right) \\
& +\tilde{\delta}_{(0,1)}((\Lambda, E)((\alpha, f),(\beta, g))) \\
= & \pi \circ \#{ }_{(\Lambda, E)} .
\end{aligned}
$$

Compare equation (2.11) with the above expression of the Lie algebroid bracket $\llbracket, \rrbracket_{(\Lambda, E)}$. ii) Let $(A, \llbracket, \rrbracket, \rho)$ be a Lie algebroid over $M$ and $\phi_{0} \in \Gamma\left(A^{*}\right)$ be a 1-cocycle. The homomorphism of $C^{\infty}(M, \mathbb{R})$-modules

$$
\begin{aligned}
\left(\rho, \phi_{0}\right): \Gamma(A) & \rightarrow \mathfrak{X}(M) \times C^{\infty}(M, \mathbb{R}) \\
X & \mapsto\left(\rho(X), \phi_{0}(X)\right),
\end{aligned}
$$


induces a homomorphism between the Lie algebroids $(A, \llbracket, \rrbracket, \rho)$ and $(T M \times \mathbb{R},[],, \pi)$, that is,

$$
\left(\rho, \phi_{0}\right) \llbracket X, Y \rrbracket=\left[\left(\rho, \phi_{0}\right)(X),\left(\rho, \phi_{0}\right)(Y)\right], \quad \pi\left(\left(\rho, \phi_{0}\right)(X)\right)=\rho(X),
$$

for $X, Y \in \Gamma(A)$. Moreover, if $\left(\rho, \phi_{0}\right)^{*}: \Omega^{1}(M) \times C^{\infty}(M, \mathbb{R}) \rightarrow \Gamma\left(A^{*}\right)$ is the adjoint homomorphism of $\left(\rho, \phi_{0}\right)$, then $\left(\rho, \phi_{0}\right)^{*}(0,1)=\phi_{0}$. As a consequence, the corresponding homomorphism $\left(\rho, \phi_{0}\right)^{k}: \Gamma\left(\wedge^{k} A\right) \rightarrow \Gamma\left(\wedge^{k}(T M \times \mathbb{R})\right) \cong \mathcal{V}^{k}(M) \oplus \mathcal{V}^{k-1}(M)$ and its adjoint homomorphism $\left(\left(\rho, \phi_{0}\right)^{k}\right)^{*}: \Omega^{k}(M) \oplus \Omega^{k-1}(M) \rightarrow \Gamma\left(\wedge^{k} A^{*}\right)$ satisfy

$$
\begin{gathered}
\left(\left(\rho, \phi_{0}\right)^{k+1}\right)^{*}(\tilde{\delta}(\alpha, \beta))=d\left(\left(\left(\rho, \phi_{0}\right)^{k}\right)^{*}(\alpha, \beta)\right), \\
\left(\left(\rho, \phi_{0}\right)^{k+1}\right)^{*}\left(\tilde{\delta}_{(0,1)}(\alpha, \beta)\right)=d_{\phi_{0}}\left(\left(\left(\rho, \phi_{0}\right)^{k}\right)^{*}(\alpha, \beta)\right) .
\end{gathered}
$$

In particular,

$$
\left(\rho, \phi_{0}\right)^{*}\left(\tilde{\delta}_{(0,1)} f\right)=\left(\rho, \phi_{0}\right)^{*}(\delta f, f)=d_{\phi_{0}} f, \text { for } f \in C^{\infty}(M, \mathbb{R}) .
$$

2.- The Lie algebroid $\left(T^{*} M \times \mathbb{R}, \llbracket, \rrbracket_{(\Lambda, E)}, \widetilde{\#}_{(\Lambda, E)}\right)$ associated with a Jacobi manifold $(M, \Lambda, E)$ Let $(M, \Lambda, E)$ be a Jacobi manifold and $\left(T^{*} M \times \mathbb{R}, \llbracket, \rrbracket_{(\Lambda, E)}, \widetilde{\#}_{(\Lambda, E)}\right)$ the associated Lie algebroid (see Section 2.2). Denote by $d_{*}$ the differential of $\left(T^{*} M \times \mathbb{R}, \llbracket, \rrbracket_{(\Lambda, E),}, \widetilde{\#}_{(\Lambda, E)}\right)$. From (2.1) and (2.10), it follows that $X_{0}=(-E, 0) \in \mathfrak{X}(M) \times C^{\infty}(M, \mathbb{R}) \cong \Gamma(T M \times \mathbb{R})$ is a 1-cocycle. Using (2.10) and (3.2), we obtain the following expression for the $X_{0^{-}}$ differential $d_{* X_{0}}=d_{*(-E, 0)}$,

$$
\begin{aligned}
d_{*(-E, 0)}(P, Q)= & (-[\Lambda, P]+(k-1) E \wedge P+\Lambda \wedge Q, \\
& {[\Lambda, Q]-(k-2) E \wedge Q+[E, P]), }
\end{aligned}
$$

for $(P, Q) \in \mathcal{V}^{k}(M) \oplus \mathcal{V}^{k-1}(M)$. Note that $d_{*(-E, 0)}$ is just the cohomology operator of the 1-differentiable Chevalley-Eilenberg cohomology complex of $M$ (see [9, 20]).

\section{$3.2 \phi_{0}$-Schouten bracket}

In [1], a skew-symmetric Schouten bracket was defined for two multilinear maps of a commutative associative algebra $\mathfrak{F}$ over $\mathbb{R}$ with unit as follows. Let $\mathcal{P}$ and $\mathcal{P}^{\prime}$ be skewsymmetric multilinear maps of degree $k$ and $k^{\prime}$, respectively, and $f_{1}, \ldots, f_{k+k^{\prime}-1} \in \mathfrak{F}$. If $A$ is any subset of $\left\{1,2, \ldots,\left(k+k^{\prime}-1\right)\right\}$, let $A^{\prime}$ denote its complement and $|A|$ the number of elements in $A$. If $|A|=l$ and the elements in $A$ are $\left\{i_{1}, \ldots, i_{l}\right\}$ in increasing order, let us write $f_{A}$ for the ordered $k$-uple $\left(f_{i_{1}}, \ldots, f_{i_{l}}\right)$. Furthermore, we write $\varepsilon_{A}$ for the sign of the permutation which rearranges the elements of the ordered $\left(k+k^{\prime}-1\right)$-uple $\left(\mathrm{A}^{\prime}, \mathrm{A}\right)$, in the original order. Then, the Schouten bracket of $\mathcal{P}$ and $\mathcal{P}^{\prime},\left[\mathcal{P}, \mathcal{P}^{\prime}\right]_{(0,1)}$, is the skew-symmetric multilinear map of degree $k+k^{\prime}-1$ given by

$$
\left[\mathcal{P}, \mathcal{P}^{\prime}\right]_{(0,1)}\left(f_{1}, \ldots f_{k+k^{\prime}-1}\right)=\sum_{|A|=k^{\prime}} \varepsilon_{A} \mathcal{P}\left(\mathcal{P}^{\prime}\left(f_{A}\right), f_{A^{\prime}}\right)+(-1)^{k k^{\prime}} \sum_{|B|=k} \varepsilon_{B} \mathcal{P}^{\prime}\left(\mathcal{P}\left(f_{B}\right), f_{B^{\prime}}\right)
$$


One can prove that if $\mathcal{P}$ and $\mathcal{P}^{\prime}$ are first-order differential operators on each of its arguments, so is $\left[\mathcal{P}, \mathcal{P}^{\prime}\right]_{(0,1)}$. In particular, if $M$ is a differentiable manifold and $\mathfrak{F}=C^{\infty}(M, \mathbb{R})$, we know that a $k$-linear skew-symmetric first-order differential operator can be identified with a pair $(P, Q) \in \mathcal{V}^{k}(M) \oplus \mathcal{V}^{k-1}(M)$ (that is, a $k$-section of $T M \times \mathbb{R} \rightarrow M$ ) in such a way that

$$
(P, Q)\left(f_{1}, \ldots, f_{k}\right)=P\left(d f_{1}, \ldots, d f_{k}\right)+\sum_{i=1}^{k}(-1)^{i+1} f_{i} Q\left(d f_{1}, \ldots, \hat{d f_{i}}, \ldots, d f_{k}\right),
$$

for $f_{1}, \ldots, f_{k} \in C^{\infty}(M, \mathbb{R})$. Under the above identification, we have that

$$
\begin{aligned}
{\left[(P, Q),\left(P^{\prime}, Q^{\prime}\right)\right]_{(0,1)}=} & \left(\left[P, P^{\prime}\right]+(-1)^{k+1}(k-1) P \wedge Q^{\prime}-\left(k^{\prime}-1\right) Q \wedge P^{\prime}\right. \\
& \left.(-1)^{k+1}\left[P, Q^{\prime}\right]-\left[Q, P^{\prime}\right]+(-1)^{k+1}\left(k-k^{\prime}\right) Q \wedge Q^{\prime}\right)
\end{aligned}
$$

for $(P, Q) \in \mathcal{V}^{k}(M) \oplus \mathcal{V}^{k-1}(M)$ and $\left(P^{\prime}, Q^{\prime}\right) \in \mathcal{V}^{k^{\prime}}(M) \oplus \mathcal{V}^{k^{\prime}-1}(M)$. If [, ] is the Schouten bracket of the Lie algebroid $(T M \times \mathbb{R},[],, \pi)$, an easy computation, using (2.4), (2.7) and (3.16), shows that

$$
\begin{aligned}
{\left[(P, Q),\left(P^{\prime}, Q^{\prime}\right)\right]_{(0,1)}=} & {\left[(P, Q),\left(P^{\prime}, Q^{\prime}\right)\right]+(-1)^{k+1}(k-1)(P, Q) \wedge\left(i_{(0,1)}\left(P^{\prime}, Q^{\prime}\right)\right) } \\
& -\left(k^{\prime}-1\right)\left(i_{(0,1)}(P, Q)\right) \wedge\left(P^{\prime}, Q^{\prime}\right) .
\end{aligned}
$$

Remark 3.4 i) Note that $(\Lambda, E) \in \Gamma\left(\wedge^{2}(T M \times \mathbb{R})\right)$ defines a Jacobi structure on $M$ if and only if $[(\Lambda, E),(\Lambda, E)]_{(0,1)}=0$ (see (2.1) and (3.16)).

ii) Using (3.15) and (3.16), we have that the $X_{0}$-differential $d_{* X_{0}}=d_{*(-E, 0)}$ of the Lie algebroid associated with a Jacobi manifold $(M, \Lambda, E)$ is given by

$$
d_{*(-E, 0)}(P, Q)=-[(\Lambda, E),(P, Q)]_{(0,1)},
$$

for $(P, Q) \in \mathcal{V}^{k}(M) \oplus \mathcal{V}^{k-1}(M)$. Compare equation (3.18) with the expression of the differential of the Lie algebroid associated with a Poisson manifold (see Section 2.2).

Suggested by (3.17), we prove the following result

Theorem 3.5 Let $(A, \llbracket, \rrbracket, \rho)$ be a Lie algebroid and $\phi_{0} \in \Gamma\left(A^{*}\right)$ a 1-cocycle. Then, there exists a unique operation $\llbracket, \rrbracket_{\phi_{0}}: \Gamma\left(\wedge^{k} A\right) \times \Gamma\left(\wedge^{k^{\prime}} A\right) \rightarrow \Gamma\left(\wedge^{k+k^{\prime}-1} A\right)$ such that

$$
\begin{gathered}
\llbracket X, f \rrbracket_{\phi_{0}}=\rho_{\phi_{0}}(X)(f), \\
\llbracket X, Y \rrbracket_{\phi_{0}}=\llbracket X, Y \rrbracket, \\
\llbracket P, P^{\prime} \rrbracket_{\phi_{0}}=(-1)^{k k^{\prime}} \llbracket P^{\prime}, P \rrbracket_{\phi_{0}}, \\
\llbracket P, P^{\prime} \wedge P^{\prime \prime} \rrbracket_{\phi_{0}}=\llbracket P, P^{\prime} \rrbracket_{\phi_{0}} \wedge P^{\prime \prime}+(-1)^{k^{\prime}(k+1)} P^{\prime} \wedge \llbracket P, P^{\prime \prime} \rrbracket_{\phi_{0}}-\left(i_{\phi_{0}} P\right) \wedge P^{\prime} \wedge P^{\prime \prime},
\end{gathered}
$$


for $f \in C^{\infty}(M, \mathbb{R}), X, Y \in \Gamma(A), P \in \Gamma\left(\wedge^{k} A\right), P^{\prime} \in \Gamma\left(\wedge^{k^{\prime}} A\right)$ and $P^{\prime \prime} \in \Gamma\left(\wedge^{k^{\prime \prime}} A\right)$. This operation is given by the general formula

$$
\llbracket P, P^{\prime} \rrbracket_{\phi_{0}}=\llbracket P, P^{\prime} \rrbracket+(-1)^{k+1}(k-1) P \wedge\left(i_{\phi_{0}} P^{\prime}\right)-\left(k^{\prime}-1\right)\left(i_{\phi_{0}} P\right) \wedge P^{\prime} .
$$

Furthermore, it satisfies the graded Jacobi identity

$$
(-1)^{k k^{\prime \prime}} \llbracket \llbracket P, P^{\prime} \rrbracket_{\phi_{0}}, P^{\prime \prime} \rrbracket_{\phi_{0}}+(-1)^{k^{\prime} k^{\prime \prime}} \llbracket \llbracket P^{\prime \prime}, P \rrbracket_{\phi_{0}}, P^{\prime} \rrbracket_{\phi_{0}}+(-1)^{k k^{\prime}} \llbracket \llbracket P^{\prime}, P^{\prime \prime} \rrbracket_{\phi_{0}}, P \rrbracket_{\phi_{0}}=0 .
$$

Proof: We define the operation $\llbracket, \rrbracket_{\phi_{0}}: \Gamma\left(\wedge^{k} A\right) \times \Gamma\left(\wedge^{k^{\prime}} A\right) \rightarrow \Gamma\left(\wedge^{k+k^{\prime}-1} A\right)$ by

$$
\llbracket P, P^{\prime} \rrbracket_{\phi_{0}}=\llbracket P, P^{\prime} \rrbracket+(-1)^{k+1}(k-1) P \wedge\left(i_{\phi_{0}} P^{\prime}\right)-\left(k^{\prime}-1\right)\left(i_{\phi_{0}} P\right) \wedge P^{\prime} .
$$

Using (3.24) and properties of the Schouten bracket of multi-sections of $A$, we deduce (3.19), (3.20), (3.21) and (3.22).

To prove the graded Jacobi identitity, we proceed as follows. If $\alpha \in \Gamma\left(A^{*}\right)$ is a 1-cocycle, we have that

$$
i_{\alpha} \llbracket X, P^{\prime} \rrbracket=\llbracket X, i_{\alpha} P^{\prime} \rrbracket-i_{d(\alpha(X))} P^{\prime},
$$

for $X \in \Gamma(A)$ and $P^{\prime} \in \Gamma\left(\wedge^{k^{\prime}} A\right)$. Using this relation and the fact that

$$
\llbracket X_{1} \wedge \ldots X_{k}, P^{\prime} \rrbracket=\sum_{i=1}^{k}(-1)^{i+1} X_{1} \wedge \ldots \wedge \hat{X}_{i} \wedge \ldots \wedge X_{k} \wedge \llbracket X_{i}, P^{\prime} \rrbracket
$$

it follows that

$$
i_{\alpha} \llbracket P, P^{\prime} \rrbracket=-\llbracket i_{\alpha} P, P^{\prime} \rrbracket+(-1)^{k+1} \llbracket P, i_{\alpha} P^{\prime} \rrbracket,
$$

for $P \in \Gamma\left(\wedge^{k} A\right)$. From (3.24) and (3.25), we deduce that

$$
i_{\phi_{0}}\left(\llbracket P, P^{\prime} \rrbracket_{\phi_{0}}\right)=-\llbracket i_{\phi_{0}} P, P^{\prime} \rrbracket_{\phi_{0}}+(-1)^{k+1} \llbracket P, i_{\phi_{0}} P^{\prime} \rrbracket_{\phi_{0}} .
$$

On the other hand, we have that

$$
\llbracket P, f \rrbracket_{\phi_{0}}=i_{d_{\phi_{0}}} P
$$

for $f \in C^{\infty}(M, \mathbb{R})$. From (3.25), (3.26) and (3.27), we obtain that

$$
\llbracket f, \llbracket P^{\prime}, P^{\prime \prime} \rrbracket_{\phi_{0}} \rrbracket_{\phi_{0}}+\llbracket \llbracket f, P^{\prime} \rrbracket_{\phi_{0}}, P^{\prime \prime} \rrbracket_{\phi_{0}}+(-1)^{k^{\prime}} \llbracket P^{\prime}, \llbracket f, P^{\prime \prime} \rrbracket_{\phi_{0}} \rrbracket_{\phi_{0}}=0 .
$$

This proves (3.23) for $k=0$.

On the other hand if $X \in \Gamma(A)$, using (3.25) and the properties of the Schouten bracket $\llbracket, \rrbracket$, it follows that

$$
\llbracket X, \llbracket P^{\prime}, P^{\prime \prime} \rrbracket_{\phi_{0}} \rrbracket_{\phi_{0}}=\llbracket \llbracket X, P^{\prime} \rrbracket_{\phi_{0}}, P^{\prime \prime} \rrbracket_{\phi_{0}}+\llbracket P^{\prime}, \llbracket X, P^{\prime \prime} \rrbracket_{\phi_{0}} \rrbracket_{\phi_{0}} .
$$

We must show that (3.23) holds, for $k \geq 1$. But, this is equivalent to prove that (3.23) holds for $P^{\prime} \in \Gamma\left(\wedge^{k^{\prime}} A\right), P^{\prime \prime} \in \Gamma\left(\wedge^{k^{\prime \prime}} A\right)$ and $P=\bar{P} \wedge Y$, with $\bar{P} \in \Gamma\left(\wedge^{k-1} A\right)$ and $Y \in \Gamma(A)$. 
We will proceed by induction on $k$. From (3.29), we deduce that the result is true for $k=1$. Now, assume that

$$
\begin{gathered}
(-1)^{(\bar{k}+1) k^{\prime \prime}} \llbracket \llbracket \bar{Q} \wedge Y, P^{\prime} \rrbracket_{\phi_{0}}, P^{\prime \prime} \rrbracket_{\phi_{0}}+(-1)^{k^{\prime} k^{\prime \prime}} \llbracket \llbracket P^{\prime \prime}, \bar{Q} \wedge Y \rrbracket_{\phi_{0}}, P^{\prime} \rrbracket_{\phi_{0}}+ \\
(-1)^{(\bar{k}+1) k^{\prime}} \llbracket \llbracket P^{\prime}, P^{\prime \prime} \rrbracket_{\phi_{0}}, \bar{Q} \wedge Y \rrbracket_{\phi_{0}}=0
\end{gathered}
$$

for $\bar{Q} \in \Gamma\left(\wedge^{\bar{k}} A\right)$, with $\bar{k} \leq k-2$.

Then, we have that

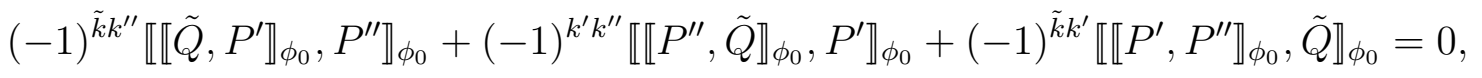

for $\tilde{Q} \in \Gamma\left(\wedge^{\tilde{k}} A\right)$, with $\tilde{k} \leq k-1$.

Using this fact, (3.26) and (3.29), we conclude that

$$
\begin{gathered}
(-1)^{k k^{\prime \prime}} \llbracket \llbracket \bar{P} \wedge Y, P^{\prime} \rrbracket_{\phi_{0}}, P^{\prime \prime} \rrbracket_{\phi_{0}}+(-1)^{k^{\prime} k^{\prime \prime}} \llbracket \llbracket P^{\prime \prime}, \bar{P} \wedge Y \rrbracket_{\phi_{0}}, P^{\prime} \rrbracket_{\phi_{0}}+ \\
(-1)^{k k^{\prime}} \llbracket \llbracket P^{\prime}, P^{\prime \prime} \rrbracket_{\phi_{0}}, \bar{P} \wedge Y \rrbracket_{\phi_{0}}=0 .
\end{gathered}
$$

Finally, if $\llbracket, \rrbracket^{\sim}: \Gamma\left(\wedge^{k} A\right) \times \Gamma\left(\wedge^{k^{\prime}} A\right) \rightarrow \Gamma\left(\wedge^{k+k^{\prime}-1} A\right)$ is an operation which satisfies (3.19), $(3.20),(3.21)$ and $(3.22)$, then it is clear that $\llbracket, \rrbracket^{\sim}=\llbracket, \rrbracket_{\phi_{0}} . \quad[Q E D$

The operation $\llbracket, \rrbracket_{\phi_{0}}$ is called the $\phi_{0}$-Schouten bracket of $(A, \llbracket, \rrbracket, \rho)$. Now, if $X \in \Gamma(A)$ and $P \in \Gamma\left(\wedge^{k} A\right)$, we can define the $\phi_{0}$-Lie derivative of $P$ by $X$ as follows

$$
\left(\mathcal{L}_{\phi_{0}}\right)_{X}(P)=\llbracket X, P \rrbracket_{\phi_{0}}
$$

From Theorem 3.5, we deduce

Proposition 3.6 Let $(A, \llbracket, \rrbracket, \rho)$ be a Lie algebroid and $\phi_{0} \in \Gamma\left(A^{*}\right)$ a 1-cocycle. If $f \in$ $C^{\infty}(M, \mathbb{R}), X \in \Gamma(A), P \in \Gamma\left(\wedge^{k} A\right)$ and $P^{\prime} \in \Gamma\left(\wedge^{k^{\prime}} A\right)$, we have

$$
\begin{aligned}
\left(\mathcal{L}_{\phi_{0}}\right)_{X}\left(P \wedge P^{\prime}\right)= & \left(\mathcal{L}_{\phi_{0}}\right)_{X}(P) \wedge P^{\prime}+P \wedge\left(\mathcal{L}_{\phi_{0}}\right)_{X}\left(P^{\prime}\right)-\phi_{0}(X) P \wedge P^{\prime} \\
& \left(\mathcal{L}_{\phi_{0}}\right)_{f X}(P)=f\left(\mathcal{L}_{\phi_{0}}\right)_{X}(P)-X \wedge i_{d f} P .
\end{aligned}
$$

Using (3.5), (3.20) (3.30) and (3.31), we obtain that

$$
\left(\mathcal{L}_{\phi_{0}}\right)_{X}\left(i_{\omega} P\right)=i_{P}\left(\left(\mathcal{L}_{\phi_{0}}\right)_{X} \omega\right)+i_{\omega}\left(\left(\mathcal{L}_{\phi_{0}}\right)_{X} P\right)+(k-1) \phi_{0}(X) i_{\omega} P
$$

for $\omega \in \Gamma\left(\wedge^{k} A^{*}\right), P \in \Gamma\left(\wedge^{k} A\right)$ and $X \in \Gamma(A)$.

Now, suppose that $(M, \Lambda, E)$ is a Jacobi manifold. We consider the 1-jet Lie algebroid $\left(T^{*} M \times \mathbb{R}, \llbracket, \rrbracket_{(\Lambda, E)}, \widetilde{\#}_{(\Lambda, E)}\right)$ associated with the Jacobi structure $(\Lambda, E)$ and the 1-cocycle $(-E, 0) \in \Gamma(T M \times \mathbb{R}) \cong \mathfrak{X}(M) \times C^{\infty}(M, \mathbb{R})$. As we know, the dual bundle $T M \times \mathbb{R}$ admits a Lie algebroid structure $([],, \pi)$ and the pair $(0,1) \in \Gamma\left(T^{*} M \times \mathbb{R}\right) \cong \Omega^{1}(M) \times C^{\infty}(M, \mathbb{R})$ is a 1-cocycle (see Sections 2.2 and 3.1).

For the above Lie algebroids and 1-cocycles, we deduce 
Proposition $3.7 \quad$ i) If $(X, f),(Y, g) \in \Gamma(T M \times \mathbb{R}) \cong \mathfrak{X}(M) \times C^{\infty}(M, \mathbb{R})$, then

$$
d_{*(-E, 0)}[(X, f),(Y, g)]=\left[(X, f), d_{*(-E, 0)}(Y, g)\right]_{(0,1)}-\left[(Y, g), d_{*(-E, 0)}(X, f)\right]_{(0,1)} .
$$

ii) If $\mathcal{L}_{*}$ denotes the Lie derivative on the Lie algebroid $\left(T^{*} M \times \mathbb{R}, \llbracket, \rrbracket_{(\Lambda, E)}\right.$, $\left.\tilde{H}_{(\Lambda, E)}\right)$, then

$$
\begin{aligned}
& \left(\mathcal{L}_{*(-E, 0)}\right)_{(0,1)}(P, Q)+\left(\mathcal{L}_{(0,1)}\right)_{(-E, 0)}(P, Q)=0, \\
& \text { for }(P, Q) \in \Gamma\left(\wedge^{k}(T M \times \mathbb{R})\right) \cong \mathcal{V}^{k}(M) \oplus \mathcal{V}^{k-1}(M) .
\end{aligned}
$$

Proof: i) It follows from (3.18), (3.21) and (3.23).

ii) Using (2.4), (3.7), (3.15), (3.16) and (3.30), we have that

$$
\begin{aligned}
\left(\mathcal{L}_{*(-E, 0)}\right)_{(0,1)}(P, Q)+\left(\mathcal{L}_{(0,1)}\right)_{(-E, 0)}(P, Q)= & \\
= & d_{*(-E, 0)}(Q, 0)+i_{(0,1)}(-[\Lambda, P]+(k-1) E \wedge P+\Lambda \wedge Q,[\Lambda, Q] \\
& -(k-2) E \wedge Q+[E, P])-([E, P],[E, Q])=0 .
\end{aligned}
$$

Remark $3.8 i)$ Let $(A, \llbracket, \rrbracket, \rho)$ be a Lie algebroid and $A^{*}$ the dual bundle to $A$. Suppose that $\left(\llbracket, \rrbracket_{*}, \rho_{*}\right)$ is a Lie algebroid structure on $A^{*}$. Then, the pair $\left(A, A^{*}\right)$ is said to be a Lie bialgebroid if

$$
d_{*} \llbracket X, Y \rrbracket=\llbracket X, d_{*} Y \rrbracket-\llbracket Y, d_{*} X \rrbracket,
$$

for $X, Y \in \Gamma(A)$, where $d_{*}$ is the differential of the Lie algebroid $\left(A^{*}, \llbracket, \rrbracket_{*}, \rho_{*}\right)$ (see [15, 23]).

ii) Let $(M, \Lambda)$ be a Poisson manifold and $\left(T^{*} M, \llbracket, \rrbracket_{\Lambda}, \#_{\Lambda}\right)$ the associated Lie algebroid (see Section 2.2). If on $T M$ we consider the trivial Lie algebroid structure, the pair $\left(T M, T^{*} M\right)$ is a Lie bialgebroid (see [23]).

iii) If $(M, \Lambda, E)$ is a Jacobi manifold and on $T M \times \mathbb{R}\left(\right.$ resp. $\left.T^{*} M \times \mathbb{R}\right)$ we consider the Lie algebroid structure $([],, \pi)$ (resp. $\left.\left(\llbracket, \rrbracket_{(\Lambda, E)}, \widetilde{\#}_{(\Lambda, E)}\right)\right)$ then, from Proposition 3.7 , we deduce that the pair $\left(T M \times \mathbb{R}, T^{*} M \times \mathbb{R}\right)$ is not, in general, a Lie bialgebroid (see [29]).

\section{Generalized Lie bialgebroids}

\subsection{Generalized Lie bialgebroids and Jacobi structures on the base space}

Let $A$ be a vector bundle over $M$ and $A^{*}$ the dual bundle to $A$. Suppose that $(\llbracket, \rrbracket, \rho)$ (resp. $\left.\left(\llbracket, \rrbracket_{*}, \rho_{*}\right)\right)$ is a Lie algebroid structure on $A$ (resp. $A^{*}$ ) and that $\phi_{0} \in \Gamma\left(A^{*}\right)$ (resp. $\left.X_{0} \in \Gamma(A)\right)$ is a 1-cocycle in the corresponding Lie algebroid cohomology complex with trivial coefficients. Then, we will use the following notation: 
- $d\left(\operatorname{resp} . d_{*}\right)$ is the differential of $(A, \llbracket \mathbb{}, \rrbracket, \rho)\left(\operatorname{resp} .\left(A^{*}, \llbracket, \rrbracket *, \rho_{*}\right)\right)$.

- $d_{\phi_{0}}$ (resp. $d_{* X_{0}}$ ) is the $\phi_{0}$-differential (resp. $X_{0}$-differential) of $A$ (resp. $A^{*}$ ).

- $\mathcal{L}\left(\right.$ resp. $\left.\mathcal{L}_{*}\right)$ is the Lie derivative of $A$ (resp. $\left.A^{*}\right)$.

- $\mathcal{L}_{\phi_{0}}$ (resp. $\mathcal{L}_{* X_{0}}$ ) is the $\phi_{0}$-Lie derivative (resp. $X_{0}$-Lie derivative).

- $\llbracket, \rrbracket_{\phi_{0}}$ (resp. $\llbracket, \rrbracket_{* X_{0}}$ ) is the $\phi_{0}$-Schouten bracket (resp. $X_{0^{-}}$Schouten bracket) on $(A, \llbracket, \rrbracket, \rho)\left(\operatorname{resp} .\left(A^{*}, \llbracket, \rrbracket *, \rho_{*}\right)\right)$.

- $\rho_{\phi_{0}}: \Gamma(A) \times C^{\infty}(M, \mathbb{R}) \rightarrow C^{\infty}(M, \mathbb{R})\left(\operatorname{resp} . \rho_{* X_{0}}: \Gamma\left(A^{*}\right) \times C^{\infty}(M, \mathbb{R}) \rightarrow C^{\infty}(M, \mathbb{R})\right)$ is the representation given by (3.1).

- $\left(\rho, \phi_{0}\right): \Gamma(A) \rightarrow \mathfrak{X}(M) \times C^{\infty}(M, \mathbb{R})\left(\right.$ resp. $\left.\left(\rho_{*}, X_{0}\right): \Gamma\left(A^{*}\right) \rightarrow \mathfrak{X}(M) \times C^{\infty}(M, \mathbb{R})\right)$ is the homomorphism of $C^{\infty}(M, \mathbb{R})$-modules given by (3.12) and $\left(\rho, \phi_{0}\right)^{*}: \Omega^{1}(M) \times C^{\infty}(M, \mathbb{R}) \rightarrow$ $\Gamma\left(A^{*}\right)$ (resp. $\left.\left(\rho_{*}, X_{0}\right)^{*}: \Omega^{1}(M) \times C^{\infty}(M, \mathbb{R}) \rightarrow \Gamma(A)\right)$ is the adjoint operator of $\left(\rho, \phi_{0}\right)$ (resp. $\left.\left(\rho_{*}, X_{0}\right)\right)$.

Definition 4.1 The pair $\left(\left(A, \phi_{0}\right),\left(A^{*}, X_{0}\right)\right)$ is said to be a generalized Lie bialgebroid over $M$ if for all $X, Y \in \Gamma(A)$ and $P \in \Gamma\left(\wedge^{k} A\right)$

$$
\begin{gathered}
d_{* X_{0}} \llbracket X, Y \rrbracket=\llbracket X, d_{* X_{0}} Y \rrbracket_{\phi_{0}}-\llbracket Y, d_{* X_{0}} X \rrbracket_{\phi_{0}}, \\
\left(\mathcal{L}_{* X_{0}}\right)_{\phi_{0}} P+\left(\mathcal{L}_{\phi_{0}}\right)_{X_{0}} P=0 .
\end{gathered}
$$

Using (3.1), (3.5), (3.20), (3.30) and (3.31), we obtain that (4.2) holds if and only if

$$
\begin{gathered}
\phi_{0}\left(X_{0}\right)=0, \quad \rho\left(X_{0}\right)=-\rho_{*}\left(\phi_{0}\right), \\
\left(\mathcal{L}_{*}\right)_{\phi_{0}} X+\llbracket X_{0}, X \rrbracket=0, \text { for } X \in \Gamma(A) .
\end{gathered}
$$

Note that (4.3) and (4.4) follow applying (4.2) to $P=f \in C^{\infty}(M, \mathbb{R})=\Gamma\left(\wedge^{0} A\right)$ and $P=X \in \Gamma(A)$.

Examples $4.2 \quad$ i) In the particular case when $\phi_{0}=0$ and $X_{0}=0$, (4.1) and (4.2) are equivalent to the condition

$$
d_{*} \llbracket X, Y \rrbracket=\llbracket X, d_{*} Y \rrbracket-\llbracket Y, d_{*} X \rrbracket .
$$

Thus, the pair $\left((A, 0),\left(A^{*}, 0\right)\right)$ is a generalized Lie bialgebroid if and only if the pair $\left(A, A^{*}\right)$ is a Lie bialgebroid.

ii) Let $(M, \Lambda, E)$ be a Jacobi manifold. From Proposition 3.7, we deduce that $((T M \times$ $\left.\mathbb{R},(0,1)),\left(T^{*} M \times \mathbb{R},(-E, 0)\right)\right)$ is a generalized Lie bialgebroid.

Next, we will show that if $\left(\left(A, \phi_{0}\right),\left(A^{*}, X_{0}\right)\right)$ is a generalized Lie bialgebroid over $M$, then $M$ carries an induced Jacobi structure. First, we will prove some results. 
Proposition 4.3 Let $\left(\left(A, \phi_{0}\right),\left(A^{*}, X_{0}\right)\right)$ be a generalized Lie bialgebroid. Then,

$$
\left(\mathcal{L}_{* X_{0}}\right)_{d_{\phi_{0}} f} X=\llbracket X, d_{* X_{0}} f \rrbracket,
$$

for $X \in \Gamma(A)$ and $f \in C^{\infty}(M, \mathbb{R})$.

Proof: Using (3.4) and the derivation law on Lie algebroids, we obtain that

$$
\begin{aligned}
d_{* X_{0}}(\llbracket X, f Y \rrbracket)= & \left(d_{* X_{0}} f\right) \wedge \llbracket X, Y \rrbracket+f d_{* X_{0}} \llbracket X, Y \rrbracket-f X_{0} \wedge \llbracket X, Y \rrbracket \\
& +d_{* X_{0}}(\rho(X)(f)) \wedge Y+\rho(X)(f) d_{* X_{0}} Y-\rho(X)(f) X_{0} \wedge Y,
\end{aligned}
$$

for $X, Y \in \Gamma(A)$ and $f \in C^{\infty}(M, \mathbb{R})$.

On the other hand, from (3.4), (3.31), (3.32) and (14.1), we deduce that

$$
\begin{aligned}
d_{* X_{0}}(\llbracket X, f Y \rrbracket)= & \left(\mathcal{L}_{\phi_{0}}\right)_{X}\left(d_{* X_{0}}(f Y)\right)-\left(\mathcal{L}_{\phi_{0}}\right)_{f Y}\left(d_{* X_{0}}(X)\right) \\
= & \left(\left(\mathcal{L}_{\phi_{0}}\right)_{X}\left(d_{* X_{0}} f\right)\right) \wedge Y+\left(d_{* X_{0}} f\right) \wedge\left(\mathcal{L}_{\phi_{0}}\right)_{X} Y-\phi_{0}(X)\left(d_{* X_{0}} f\right) \wedge Y \\
& +f\left(\mathcal{L}_{\phi_{0}}\right)_{X}\left(d_{* X_{0}} Y\right)+\rho(X)(f) d_{* X_{0}} Y \\
& -f\left(\left(\mathcal{L}_{\phi_{0}}\right)_{X} X_{0} \wedge Y+X_{0} \wedge\left(\mathcal{L}_{\phi_{0}}\right)_{X} Y-\phi_{0}(X) X_{0} \wedge Y\right) \\
& -\rho(X)(f) X_{0} \wedge Y-f\left(\mathcal{L}_{\phi_{0}}\right)_{Y}\left(d_{* X_{0}} X\right)-i_{d f}\left(d_{* X_{0}} X\right) \wedge Y .
\end{aligned}
$$

Thus, using again (4.1), it follows that

$$
\begin{aligned}
d_{* X_{0}}(\rho(X)(f)) \wedge Y= & \left(\left(\mathcal{L}_{\phi_{0}}\right)_{X} d_{* X_{0}} f-\phi_{0}(X) d_{* X_{0}} f-f\left(\mathcal{L}_{\phi_{0}}\right)_{X} X_{0}\right. \\
& \left.+f \phi_{0}(X) X_{0}-i_{d f}\left(d_{* X_{0}} X\right)\right) \wedge Y,
\end{aligned}
$$

and so

$d_{* X_{0}}(\rho(X)(f))-\left(\mathcal{L}_{\phi_{0}}\right)_{X} d_{* X_{0}} f+\phi_{0}(X) d_{* X_{0}} f+f\left(\mathcal{L}_{\phi_{0}}\right)_{X} X_{0}-f \phi_{0}(X) X_{0}+i_{d f}\left(d_{* X_{0}} X\right)=0$, which, by (3.4), (3.7) and (4.4), implies (4.5).

$Q E D$

Corollary 4.4 Under the same hypothesis as in Proposition 4.9, we have

$$
\llbracket d_{* X_{0}} g, d_{* X_{0}} f \rrbracket=d_{* X_{0}}\left(d_{\phi_{0}} f \cdot d_{* X_{0}} g\right),
$$

for all $f, g \in C^{\infty}(M, \mathbb{R})$.

Proof: If $\tilde{\delta}_{(0,1)}$ is the operator defined by (3.11) then, from (3.1), (3.5), (3.7), (3.12), (3.14) and Proposition 4.3, we have

$$
\begin{aligned}
\llbracket d_{* X_{0}} g, d_{* X_{0}} f \rrbracket & =\left(\mathcal{L}_{* X_{0}}\right)_{d_{\phi_{0}} f}\left(d_{* X_{0}} g\right)=d_{* X_{0}}\left(\left(\mathcal{L}_{* X_{0}}\right)_{d_{\phi_{0}} f}(g)\right) \\
& =d_{* X_{0}}\left(\rho_{* X_{0}}\left(d_{\phi_{0}} f\right)(g)\right)=d_{* X_{0}}\left(\tilde{\delta}_{(0,1)} g \cdot\left(\rho_{*}, X_{0}\right)\left(d_{\phi_{0}} f\right)\right) \\
& =d_{* X_{0}}\left(d_{\phi_{0}} f \cdot\left(\rho_{*}, X_{0}\right)^{*}\left(\tilde{\delta}_{(0,1)} g\right)\right)=d_{* X_{0}}\left(d_{\phi_{0}} f \cdot d_{* X_{0}} g\right) .
\end{aligned}
$$


Remark 4.5 Using (3.11), (3.12) and (3.14), it follows that

$$
\begin{aligned}
d_{\phi_{0}} f \cdot d_{* X_{0}} g & =\tilde{\delta}_{(0,1)} f \cdot\left(\left(\rho, \phi_{0}\right) \circ\left(\rho_{*}, X_{0}\right)^{*}\right)\left(\tilde{\delta}_{(0,1)} g\right) \\
& =\pi_{(0,1)}\left(\left(\left(\rho, \phi_{0}\right) \circ\left(\rho_{*}, X_{0}\right)^{*}\right)\left(\tilde{\delta}_{(0,1)} g\right), f\right),
\end{aligned}
$$

where $\pi_{(0,1)}:\left(\mathfrak{X}(M) \times C^{\infty}(M, \mathbb{R})\right) \times C^{\infty}(M, \mathbb{R}) \rightarrow C^{\infty}(M, \mathbb{R})$ is the representation given by (3.10).

Now, we will prove the main result of this section.

Theorem 4.6 Let $\left(\left(A, \phi_{0}\right),\left(A^{*}, X_{0}\right)\right)$ be a generalized Lie bialgebroid. Then, the bracket of functions $\{\}:, C^{\infty}(M, \mathbb{R}) \times C^{\infty}(M, \mathbb{R}) \rightarrow C^{\infty}(M, \mathbb{R})$ given by

$$
\{f, g\}:=-d_{\phi_{0}} f \cdot d_{* X_{0}} g, \text { for } f, g \in C^{\infty}(M, \mathbb{R}),
$$

defines a Jacobi structure on $M$.

Proof: First of all, we need to prove that $\{$,$\} is skew-symmetric. For that purpose, we$ first show that

$$
\tilde{\delta}_{(0,1)} f \cdot\left(\left(\left(\rho, \phi_{0}\right) \circ\left(\rho_{*}, X_{0}\right)^{*}\right)(0,1)\right)=-\tilde{\delta}_{(0,1)} \cdot\left(\left(\left(\rho, \phi_{0}\right) \circ\left(\rho_{*}, X_{0}\right)^{*}\right)(\delta f, 0)\right),
$$

for $f \in C^{\infty}(M, \mathbb{R})$. We have that (see (3.11), (3.12) and (4.3))

$$
\tilde{\delta}_{(0,1)} f \cdot\left(\left(\left(\rho, \phi_{0}\right) \circ\left(\rho_{*}, X_{0}\right)^{*}\right)(0,1)\right)=(\delta f, f) \cdot\left(\rho\left(X_{0}\right), 0\right)=\rho\left(X_{0}\right)(f)=-\rho_{*}\left(\phi_{0}\right)(f) .
$$

On the other hand, from (3.12) and (4.3), we deduce that

$$
\left.-\tilde{\delta}_{(0,1)} 1 \cdot\left(\left(\left(\rho, \phi_{0}\right) \circ\left(\rho_{*}, X_{0}\right)^{*}\right)(\delta f, 0)\right)=-\phi_{0} \cdot\left(\rho_{*}, X_{0}\right)^{*}(\delta f, 0)\right)=-\rho_{*}\left(\phi_{0}\right)(f) .
$$

Thus, we deduce (4.7). Using (3.11), (4.7) and Remark 4.5, we obtain that

$$
\{f, f\}=-\left(\left(\left(\rho, \phi_{0}\right) \circ\left(\rho_{*}, X_{0}\right)^{*}\right)(\delta f, 0)\right) \cdot(\delta f, 0) .
$$

Now, we will prove that

$$
\left(\left(\left(\rho, \phi_{0}\right) \circ\left(\rho_{*}, X_{0}\right)^{*}\right)(\delta f, 0)\right) \cdot(\delta f, 0)=0 .
$$

From (3.4), (4.8), Corollary 4.4 and Remark 4.5, it follows that $d_{* X_{0}}\left(\left(\rho, \phi_{0}\right) \circ\left(\rho_{*}, X_{0}\right)^{*}\right)$ $\left.\left(\delta f^{2}, 0\right) \cdot\left(\delta f^{2}, 0\right)\right)=0$. Then,

$$
\begin{aligned}
0 & =\left(\left(\left(\rho, \phi_{0}\right) \circ\left(\rho_{*}, X_{0}\right)^{*}\right)(\delta f, 0) \cdot(\delta f, 0)\right)\left(d_{* X_{0}} f^{2}-f^{2} X_{0}\right) \\
& =\left(\left(\left(\rho, \phi_{0}\right) \circ\left(\rho_{*}, X_{0}\right)^{*}\right)(\delta f, 0) \cdot(\delta f, 0)\right) d_{*} f^{2} \\
& =2 f\left(\left(\left(\rho, \phi_{0}\right) \circ\left(\rho_{*}, X_{0}\right)^{*}\right)(\delta f, 0) \cdot(\delta f, 0)\right) d_{*} f .
\end{aligned}
$$


On the other hand, in general, $d_{*} g=\left(\rho_{*}, X_{0}\right)^{*}(\delta g, 0)$. Thus, using (4.9),

$$
f\left(\left(\left(\rho, \phi_{0}\right) \circ\left(\rho_{*}, X_{0}\right)^{*}\right)(\delta f, 0) \cdot(\delta f, 0)\right)^{2}=0, \quad \text { for all } f .
$$

This implies that

$$
\left(\left(\left(\rho, \phi_{0}\right) \circ\left(\rho_{*}, X_{0}\right)^{*}\right)(\delta f, 0)\right) \cdot(\delta f, 0)=0
$$

as we wanted to prove. Therefore, we conclude that $\{f, f\}=0$, for all $f \in C^{\infty}(M, \mathbb{R})$, that is, $\{$,$\} is skew-symmetric.$

From (3.3) and (3.4), we deduce that $\{$,$\} is a first-order differential operator on each of$ its arguments.

Now, let us prove the Jacobi identity. Using (4.6), we have

$$
d_{\phi_{0}} h \cdot \llbracket d_{* X_{0}} g, d_{* X_{0}} f \rrbracket=-d_{\phi_{0}} h \cdot d_{* X_{0}}(\{f, g\}) .
$$

Thus, from (3.13) and (3.14), we deduce that

$$
\tilde{\delta}_{(0,1)} h \cdot\left[\left(\rho, \phi_{0}\right)\left(\left(\rho_{*}, X_{0}\right)^{*}\left(\tilde{\delta}_{(0,1)} g\right)\right),\left(\rho, \phi_{0}\right)\left(\left(\rho_{*}, X_{0}\right)^{*}\left(\tilde{\delta}_{(0,1)} f\right)\right)\right]=-d_{\phi_{0}} h \cdot d_{* X_{0}}(\{f, g\}),
$$

or equivalently

$\pi_{(0,1)}\left(\left[\left(\left(\rho, \phi_{0}\right) \circ\left(\rho_{*}, X_{0}\right)^{*}\right)\left(\tilde{\delta}_{(0,1)}(g)\right),\left(\left(\rho, \phi_{0}\right) \circ\left(\rho_{*}, X_{0}\right)^{*}\right)\left(\tilde{\delta}_{(0,1)}(f)\right)\right], h\right)=-d_{\phi_{0}} h \cdot d_{* X_{0}}(\{f, g\})$.

Consequently, since $\pi_{(0,1)}$ is a representation of the Lie algebra $\left(\mathfrak{X}(M) \times C^{\infty}(M, \mathbb{R}),[],\right)$ on the space $C^{\infty}(M, \mathbb{R})$, this implies that (see Remark 4.5)

$$
\{f,\{g, h\}\}+\{g,\{h, f\}\}+\{h,\{f, g\}\}=0 .
$$

$Q E D$

From (3.2) and (4.3), we have that

$$
\{f, g\}=-d f \cdot d_{*} g+f \rho\left(X_{0}\right)(g)-g \rho\left(X_{0}\right)(f),
$$

for $f, g \in C^{\infty}(M, \mathbb{R})$. Since the differential $d$ is a derivation with respect to the ordinary multiplication of functions we have that the map $(f, g) \mapsto d f \cdot d_{*} g$, for $f, g \in C^{\infty}(M, \mathbb{R})$, is also a derivation on each of its arguments. Thus, we can define the 2 -vector $\Lambda \in \mathcal{V}^{2}(M)$ characterized by the relation

$$
\Lambda(\delta f, \delta g)=-d f \cdot d_{*} g=d g \cdot d_{*} f
$$

for $f, g \in C^{\infty}(M, \mathbb{R})$, and the vector field $E \in \mathfrak{X}(M)$ by

$$
E=\rho\left(X_{0}\right)=-\rho_{*}\left(\phi_{0}\right) \text {. }
$$

From (4.10), we obtain that

$$
\{f, g\}=\Lambda(\delta f, \delta g)+f E(g)-g E(f),
$$


for $f, g \in C^{\infty}(M, \mathbb{R})$. Therefore, the pair $(\Lambda, E)$ is the Jacobi structure induced by the Jacobi bracket $\{$,$\} .$

If $\left(A, A^{*}\right)$ is a Lie bialgebroid, then the pair $\left((A, 0),\left(A^{*}, 0\right)\right)$ is a generalized Lie bialgebroid and, by Theorem 4.6, a Jacobi structure $(\Lambda, E)$ can be defined on the base space $M$. Since $\phi_{0}=X_{0}=0$, we deduce that $E=0$, that is, the Jacobi structure is Poisson, which implies a well known result (see [23]): given a Lie bialgebroid $\left(A, A^{*}\right)$ over $M$, the base space $M$ carries an induced Poisson structure.

\subsection{Lie bialgebroids and generalized Lie bialgebroids}

Along this section we will use the same notation as in the precedent one.

\subsubsection{Time-dependent sections of a Lie algebroid}

Let $(A, \llbracket, \rrbracket, \rho)$ be a Lie algebroid over $M$. Then, the space of sections $\Gamma(\tilde{A})$ of the vector bundle $\tilde{A}=A \times \mathbb{R} \rightarrow M \times \mathbb{R}$ can be identified with the set of time-dependent sections of $A \rightarrow M$. Under this identification, the Lie bracket $\llbracket$, induces, in a natural way, a Lie bracket on $\Gamma(\tilde{A})$ which is also denoted by $\llbracket$, $\rrbracket$. In fact, if $\tilde{X}, \tilde{Y} \in \Gamma(\tilde{A})$ then $\llbracket \tilde{X}, \tilde{Y} \rrbracket$ is the time-dependent section of $A \rightarrow M$ given by

$$
\llbracket \tilde{X}, \tilde{Y} \rrbracket(x, t)=\llbracket \tilde{X}_{t}, \tilde{Y}_{t} \rrbracket(x),
$$

for all $(x, t) \in M \times \mathbb{R}$, where $\tilde{X}_{t}$ and $\tilde{Y}_{t}$ are the sections of $A \rightarrow M$ defined by

$$
\tilde{X}_{t}(y)=\tilde{X}(y, t), \quad \tilde{Y}_{t}(y)=\tilde{Y}(y, t),
$$

for all $y \in M$. The anchor map $\rho: A \rightarrow T M$ induces a bundle map from $\tilde{A}$ into $T M \subseteq T(M \times \mathbb{R}) \cong T M \oplus T \mathbb{R}$ which we also denote by $\rho$. A direct computation shows that the triple $(\tilde{A}, \llbracket, \rrbracket, \rho)$ is a Lie algebroid over $M \times \mathbb{R}$.

Now, denote by $d$ the differentials of the Lie algebroids $(A, \llbracket, \rrbracket, \rho)$ and $(\tilde{A}, \llbracket, \rrbracket, \rho)$. Then, if $\tilde{\omega} \in \Gamma\left(\wedge^{k} \tilde{A}^{*}\right)$ and $(x, t) \in M \times \mathbb{R}, d \tilde{\omega} \in \Gamma\left(\wedge^{k+1} \tilde{A}^{*}\right)$ and

$$
(d \tilde{\omega})(x, t)=\left(d \tilde{\omega}_{t}\right)(x) .
$$

We also denote by $\llbracket, \rrbracket$ the Schouten bracket of the Lie algebroid $(\tilde{A}, \llbracket, \rrbracket, \rho)$.

On the other hand, for $\tilde{P} \in \Gamma\left(\wedge^{k} \tilde{A}\right)$ or $\tilde{\omega} \in \Gamma\left(\wedge^{k} \tilde{A}^{*}\right)$, one can define its derivative with respect to the time

$$
\frac{\partial \tilde{P}}{\partial t} \in \Gamma\left(\wedge^{k} \tilde{A}\right), \quad \frac{\partial \tilde{\omega}}{\partial t} \in \Gamma\left(\wedge^{k} \tilde{A}^{*}\right) .
$$

Thus, we have two $\mathbb{R}$-linear operators of degree zero

$$
\frac{\partial}{\partial t}: \Gamma\left(\wedge^{k} \tilde{A}\right) \rightarrow \Gamma\left(\wedge^{k} \tilde{A}\right), \quad \frac{\partial}{\partial t}: \Gamma\left(\wedge^{k} \tilde{A}^{*}\right) \rightarrow \Gamma\left(\wedge^{k} \tilde{A}^{*}\right),
$$


which have the following properties

$$
\begin{gathered}
\frac{\partial}{\partial t}(\tilde{P} \wedge \tilde{Q})=\frac{\partial \tilde{P}}{\partial t} \wedge \tilde{Q}+\tilde{P} \wedge \frac{\partial \tilde{Q}}{\partial t}, \quad \frac{\partial}{\partial t}(\tilde{\omega} \wedge \tilde{\mu})=\frac{\partial \tilde{\omega}}{\partial t} \wedge \tilde{\mu}+\tilde{\omega} \wedge \frac{\partial \tilde{\mu}}{\partial t} \\
\frac{\partial}{\partial t} \llbracket \tilde{P}, \tilde{Q} \rrbracket=\llbracket \frac{\partial \tilde{P}}{\partial t}, \tilde{Q} \rrbracket+\llbracket \tilde{P}, \frac{\partial \tilde{Q}}{\partial t} \rrbracket \\
d\left(\frac{\partial \tilde{\omega}}{\partial t}\right)=\frac{\partial}{\partial t}(d \tilde{\omega}),
\end{gathered}
$$

for $\tilde{P} \in \Gamma\left(\wedge^{k} \tilde{A}\right), \tilde{Q} \in \Gamma\left(\wedge^{r} \tilde{A}\right), \tilde{\omega} \in \Gamma\left(\wedge^{k} \tilde{A}^{*}\right)$ and $\tilde{\mu} \in \Gamma\left(\wedge^{r} \tilde{A}^{*}\right)$.

Next, suppose that $\phi_{0} \in \Gamma\left(A^{*}\right)$ is a 1-cocycle. In 11] we proved that the vector bundle $\tilde{A} \rightarrow M \times \mathbb{R}$ admits a Lie algebroid structure $\left(\llbracket, \rrbracket^{\wedge} \phi_{0}, \hat{\rho}^{\phi_{0}}\right)$ where, under the above identifications, $\llbracket, \rrbracket^{\wedge} \phi_{0}$ and $\hat{\rho}^{\phi_{0}}$ are defined by

$$
\begin{gathered}
\llbracket \tilde{X}, \tilde{Y} \rrbracket^{\wedge} \phi_{0}=e^{-t}\left(\llbracket \tilde{X}, \tilde{Y} \rrbracket+\phi_{0}(\tilde{X})\left(\frac{\partial \tilde{Y}}{\partial t}-\tilde{Y}\right)-\phi_{0}(\tilde{Y})\left(\frac{\partial \tilde{X}}{\partial t}-\tilde{X}\right)\right), \\
\hat{\rho}^{\phi_{0}}(\tilde{X})=e^{-t}\left(\rho(\tilde{X})+\phi_{0}(\tilde{X}) \frac{\partial}{\partial t}\right)
\end{gathered}
$$

for all $\tilde{X}, \tilde{Y} \in \Gamma(\tilde{A})$. If $\hat{d}^{\phi_{0}}$ is the differential of the Lie algebroid $\left(\tilde{A}, \llbracket, \rrbracket^{\wedge} \phi_{0}, \hat{\rho}^{\phi_{0}}\right)$, we have

$$
\begin{gathered}
\hat{d}^{\phi_{0}} \tilde{f}=e^{-t}\left(d \tilde{f}+\frac{\partial \tilde{f}}{\partial t} \phi_{0}\right), \\
\hat{d}^{\phi_{0}} \tilde{\phi}=e^{-t}\left(d_{\phi_{0}} \tilde{\phi}+\phi_{0} \wedge \frac{\partial \tilde{\phi}}{\partial t}\right),
\end{gathered}
$$

for $\tilde{f} \in C^{\infty}(M \times \mathbb{R}, \mathbb{R})$ and $\tilde{\phi} \in \Gamma\left(\tilde{A}^{*}\right)=\Gamma\left(A^{*} \times \mathbb{R}\right)$.

In a similar way, we can define the bracket $\llbracket, \rrbracket^{-\phi_{0}}$ on $\Gamma(\tilde{A})$ and the map $\bar{\rho}^{\phi_{0}}: \Gamma(\tilde{A}) \rightarrow$ $\mathfrak{X}(M \times \mathbb{R})$ by

$$
\begin{gathered}
\llbracket \tilde{X}, \tilde{Y} \rrbracket^{-\phi_{0}}=\llbracket \tilde{X}, \tilde{Y} \rrbracket+\phi_{0}(\tilde{X}) \frac{\partial \tilde{Y}}{\partial t}-\phi_{0}(\tilde{Y}) \frac{\partial \tilde{X}}{\partial t}, \\
\bar{\rho}^{\phi_{0}}(\tilde{X})=\rho(\tilde{X})+\phi_{0}(\tilde{X}) \frac{\partial}{\partial t}
\end{gathered}
$$

for all $\tilde{X}, \tilde{Y} \in \Gamma(\tilde{A})$. Moreover, the bundle map $\Psi: \tilde{A} \rightarrow \tilde{A},(v, t) \mapsto\left(e^{t} v, t\right)$, is an isomorphism of vector bundles and

$$
\hat{\rho}^{\phi_{0}} \circ \Psi=\bar{\rho}^{\phi_{0}}, \quad \Psi \llbracket \tilde{X}, \tilde{Y} \rrbracket^{-\phi_{0}}=\llbracket \Psi \tilde{X}, \Psi \tilde{Y} \rrbracket^{\wedge \phi_{0}} .
$$

Thus,

Proposition 4.7 Let $(A, \llbracket, \rrbracket, \rho)$ be a Lie algebroid and $\phi_{0} \in \Gamma\left(A^{*}\right)$ a 1-cocycle. Then:

i) The triples $\left(\tilde{A}, \llbracket, \rrbracket^{\wedge} \phi_{0}, \hat{\rho}^{\phi_{0}}\right)$ and $\left(\tilde{A}, \llbracket, \rrbracket^{-\phi_{0}}, \bar{\rho}^{\phi_{0}}\right)$ are Lie algebroids over $M \times \mathbb{R}$. 
ii) The map $\Psi: \tilde{A} \rightarrow \tilde{A}$ defines an isomorphism between the Lie algebroids $\left(\tilde{A}, \llbracket, \rrbracket^{-\phi_{0}}\right.$, $\left.\bar{\rho}^{\phi_{0}}\right)$ and $\left(\tilde{A}, \llbracket, \rrbracket^{\wedge \phi_{0}}, \hat{\rho}^{\phi_{0}}\right)$.

If $\bar{d}^{\phi_{0}}$ is the differential of the algebroid $\left(\tilde{A}, \llbracket, \rrbracket^{-\phi_{0}}, \bar{\rho}^{\phi_{0}}\right)$, we have

$$
\begin{gathered}
\bar{d}^{\phi_{0}} \tilde{f}=d \tilde{f}+\frac{\partial \tilde{f}}{\partial t} \phi_{0}, \\
\bar{d}^{\phi_{0}} \tilde{\phi}=d \tilde{\phi}+\phi_{0} \wedge \frac{\partial \tilde{\phi}}{\partial t},
\end{gathered}
$$

for $\tilde{f} \in C^{\infty}(M \times \mathbb{R}, \mathbb{R})$ and $\tilde{\phi} \in \Gamma\left(\tilde{A}^{*}\right)=\Gamma\left(A^{*} \times \mathbb{R}\right)$. Moreover,

$$
\llbracket \tilde{X}, \tilde{P} \rrbracket^{-\phi_{0}}=\llbracket \tilde{X}, \tilde{P} \rrbracket_{\phi_{0}}+\phi_{0}(\tilde{X})\left(\tilde{P}+\frac{\partial \tilde{P}}{\partial t}\right)-\frac{\partial \tilde{X}}{\partial t} \wedge i_{\phi_{0}} \tilde{P},
$$

for $\tilde{X} \in \Gamma(\tilde{A})$ and $\tilde{P} \in \Gamma\left(\wedge^{2} \tilde{A}\right)$.

Now, let $A \rightarrow M$ be a vector bundle over a manifold $M$ and suppose that $\llbracket, \rrbracket: \Gamma(A) \times$ $\Gamma(A) \rightarrow \Gamma(A)$ is a bracket on the space $\Gamma(A)$, that $\rho: \Gamma(A) \rightarrow \mathfrak{X}(M)$ is a homomorphism of $C^{\infty}(M, \mathbb{R})$-modules and that $\phi_{0}$ is a section of the dual bundle $A^{*}$.

We can define the bracket $\llbracket, \rrbracket^{-\phi_{0}}: \Gamma(\tilde{A}) \times \Gamma(\tilde{A}) \rightarrow \Gamma(\tilde{A})$ on the space $\Gamma(\tilde{A})$ and the homomorphism of $C^{\infty}(M \times \mathbb{R}, \mathbb{R})$-modules $\bar{\rho}^{\phi_{0}}: \Gamma(\tilde{A}) \rightarrow \mathfrak{X}(M \times \mathbb{R})$ given by (4.19).

Proposition 4.8 If the triple $\left(\tilde{A}, \llbracket, \rrbracket^{-\phi_{0}}, \bar{\rho}^{\phi_{0}}\right)$ is a Lie algebroid on $M \times \mathbb{R}$ then the triple $(A, \llbracket, \rrbracket, \rho)$ is a Lie algebroid on $M$ and the section $\phi_{0}$ is a 1-cocycle.

Proof: From (4.19), it follows that $\llbracket X, Y \rrbracket^{-\phi_{0}}=\llbracket X, Y \rrbracket$, for $X, Y \in \Gamma(A)$. Thus, we have that the bracket $\llbracket, \rrbracket$ defines a Lie algebra structure on $\Gamma(A)$.

On the other hand, since $\bar{\rho}^{\phi_{0}} \llbracket X, Y \rrbracket^{-\phi_{0}}=\left[\bar{\rho}^{\phi_{0}}(X), \bar{\rho}^{\phi_{0}}(Y)\right]$, we deduce that (see 44.19))

$$
\rho \llbracket X, Y \rrbracket=[\rho(X), \rho(Y)], \quad \phi_{0} \llbracket X, Y \rrbracket=\rho(X)\left(\phi_{0}(Y)\right)-\rho(Y)\left(\phi_{0}(X)\right) .
$$

Finally, if $f \in C^{\infty}(M, \mathbb{R})$ then, using (4.19) and the fact that $\llbracket X, f Y \rrbracket^{-\phi_{0}}=f \llbracket X, Y \rrbracket^{-\phi_{0}}+$ $\left(\bar{\rho}^{\phi_{0}}(X)(f)\right) Y$, we obtain that

$$
\llbracket X, f Y \rrbracket=f \llbracket X, Y \rrbracket+(\rho(X)(f)) Y .
$$

From Propositions 4.7 and 4.8, we conclude

Proposition 4.9 Let $A \rightarrow M$ be a vector bundle over a manifold $M$. Suppose that $\llbracket, \rrbracket: \Gamma(A) \times \Gamma(A) \rightarrow \Gamma(A)$ is a bracket on the space $\Gamma(A)$, that $\rho: \Gamma(A) \rightarrow \mathfrak{X}(M)$ is a homomorphism of $C^{\infty}(M, \mathbb{R})$-modules and that $\phi_{0}$ is a section of the dual bundle $A^{*}$. If $\llbracket, \rrbracket^{\phi_{0}}: \Gamma(\tilde{A}) \times \Gamma(\tilde{A}) \rightarrow \Gamma(\tilde{A})$ and $\hat{\rho}^{\phi_{0}}: \Gamma(\tilde{A}) \rightarrow \mathfrak{X}(M \times \mathbb{R})$ (respectively, $\llbracket, \rrbracket^{-\phi_{0}}:$ 
$\Gamma(\tilde{A}) \times \Gamma(\tilde{A}) \rightarrow \Gamma(\tilde{A})$ and $\left.\bar{\rho}^{\phi_{0}}: \Gamma(\tilde{A}) \rightarrow \mathfrak{X}(M \times \mathbb{R})\right)$ are the bracket on $\Gamma(\tilde{A})$ and the homomorphism of $C^{\infty}(M \times \mathbb{R}, \mathbb{R})$-modules given by (4.10) (respectively, 4.19)) then the following conditions are equivalent:

i) The triple $(A, \llbracket, \rrbracket, \rho)$ is a Lie algebroid and $\phi_{0}$ is a 1-cocycle.

ii) The triple $\left(\tilde{A}, \llbracket, \rrbracket^{\wedge \phi_{0}}, \hat{\rho}^{\phi_{0}}\right)$ is a Lie algebroid.

iii) The triple $\left(\tilde{A}, \llbracket, \rrbracket^{-\phi_{0}}, \bar{\rho}^{\phi_{0}}\right)$ is a Lie algebroid.

\subsubsection{Lie bialgebroids and generalized Lie bialgebroids}

First of all, we will prove a general result which will be useful in the sequel.

Suppose that $\left(A_{i}, \llbracket, \rrbracket_{i}, \rho_{i}\right), i=1,2$, are two Lie algebroids over $M$ such that the dual bundles $A_{1}^{*}$ and $A_{2}^{*}$ are Lie algebroids with Lie algebroid structures $\left(\llbracket, \rrbracket_{1 *}, \rho_{1 *}\right)$ and $\left(\llbracket, \rrbracket_{2 *}, \rho_{2 *}\right)$, respectively.

Proposition 4.10 Let $\Phi: A_{1} \rightarrow A_{2}$ be a Lie algebroid isomorphism such that its adjoint homomorphism $\Phi^{*}: A_{2}^{*} \rightarrow A_{1}^{*}$ is also a Lie algebroid isomorphism. Then, if $\left(A_{1}, A_{1}^{*}\right)$ is a Lie bialgebroid, so is $\left(A_{2}, A_{2}^{*}\right)$.

Proof: Denote also by $\Phi: \wedge^{k} A_{1} \rightarrow \wedge^{k} A_{2}$ the isomorphism between the vector bundles $\wedge^{k} A_{1} \rightarrow M$ and $\wedge^{k} A_{2} \rightarrow M$ induced by $\Phi: A_{1} \rightarrow A_{2}$. If $\Phi: \Gamma\left(\wedge^{k} A_{1}\right) \rightarrow \Gamma\left(\wedge^{k} A_{2}\right)$ is the corresponding isomorphism of $C^{\infty}(M, \mathbb{R})$-modules, we have that

$$
\begin{gathered}
\Phi(P)\left(\psi_{1}, \ldots, \psi_{k}\right)=P\left(\Phi^{*} \psi_{1}, \ldots, \Phi^{*} \psi_{k}\right), \\
\Phi\left(X_{1} \wedge \ldots \wedge X_{k}\right)=\Phi\left(X_{1}\right) \wedge \ldots \wedge \Phi\left(X_{k}\right),
\end{gathered}
$$

for $P \in \Gamma\left(\wedge^{k} A_{1}\right), \psi_{1}, \ldots, \psi_{k} \in \Gamma\left(A_{2}^{*}\right)$ and $X_{1}, \ldots, X_{k} \in \Gamma\left(A_{1}\right)$. Thus, using that $\Phi$ and $\Phi^{*}$ are Lie algebroid isomorphisms, it follows that

$$
d_{2 *}\left(\Phi\left(X_{1}\right)\right)=\Phi\left(d_{1 *} X_{1}\right), \quad \Phi \llbracket X_{1}, P_{1} \rrbracket_{1}=\llbracket \Phi\left(X_{1}\right), \Phi\left(P_{1}\right) \rrbracket_{2},
$$

for $X_{1} \in \Gamma\left(A_{1}\right)$ and $P_{1} \in \Gamma\left(\wedge^{k} A_{1}\right)$, where $d_{1 *}\left(\right.$ resp. $\left.d_{2 *}\right)$ is the differential of $\left(A_{1}^{*}, \llbracket, \rrbracket_{1 *}\right.$, $\left.\rho_{1 *}\right)\left(\operatorname{resp} .\left(A_{2}^{*}, \llbracket, \rrbracket_{2 *}, \rho_{2 *}\right)\right)$.

Now, if $X_{2}, Y_{2} \in \Gamma\left(A_{2}\right)$ then there exist $X_{1}, Y_{1} \in \Gamma\left(A_{1}\right)$ such that $Y_{i}=\Phi\left(X_{i}\right)$, for $i=1,2$. Therefore, from (4.23) and since $\left(A_{1}, A_{1}^{*}\right)$ is a Lie bialgebroid, we obtain that

$$
\begin{aligned}
d_{2 *} \llbracket X_{2}, Y_{2} \rrbracket_{2} & =\left(d_{2 *} \circ \Phi\right) \llbracket X_{1}, Y_{1} \rrbracket_{1}=\Phi\left(\llbracket X_{1}, d_{1 *} Y_{1} \rrbracket_{1}-\llbracket Y_{1}, d_{1 *} X_{1} \rrbracket_{1}\right) \\
& =\llbracket X_{2}, d_{2 *} Y_{2} \rrbracket_{2}-\llbracket Y_{2}, d_{2 *} X_{2} \rrbracket_{2} .
\end{aligned}
$$

Consequently, $\left(A_{2}, A_{2}^{*}\right)$ is a Lie bialgebroid.

$Q E D$

Next, assume that $(M, \Lambda, E)$ is a Jacobi manifold. Consider on $A=T M \times \mathbb{R}$ and on $A^{*}=T^{*} M \times \mathbb{R}$ the Lie algebroid structures $([],, \pi)$ and $\left(\llbracket, \rrbracket_{(\Lambda, E)}, \widetilde{\#}_{(\Lambda, E)}\right)$, respectively. Then, the pair $\left(\left(A, \phi_{0}=(0,1)\right),\left(A^{*}, X_{0}=(-E, 0)\right)\right)$ is a generalized Lie bialgebroid. 
On the other hand, the map $\Phi: \tilde{A}=A \times \mathbb{R} \rightarrow T(M \times \mathbb{R})$ defined by

$$
\Phi\left(\left(v_{x_{0}}, \lambda_{0}\right), t_{0}\right)=v_{x_{0}}+\left.\lambda_{0} \frac{\partial}{\partial t}\right|_{t_{0}},
$$

for $x_{0} \in M, v_{x_{0}} \in T_{x_{0}} M$ and $\lambda_{0}, t_{0} \in \mathbb{R}$, induces an isomorphism between the vector bundles $A \times \mathbb{R} \rightarrow M \times \mathbb{R}$ and $T(M \times \mathbb{R}) \rightarrow M \times \mathbb{R}$. Thus, $\tilde{A}=A \times \mathbb{R}$ can be identified with $T(M \times \mathbb{R})$ and, under this identification, the Lie algebroid structure $\left([,]^{-\phi_{0}}, \bar{\pi}^{\phi_{0}}\right)$ is just the trivial Lie algebroid structure on $T(M \times \mathbb{R})$ (see (4.19)). Note that if $(\tilde{X}, \tilde{f})$ is a time-dependent section of the vector bundle $T M \times \mathbb{R} \rightarrow M$ then $\frac{\partial(\tilde{X}, \tilde{f})}{\partial t}$ is the timedependent section given by $\left(\left[\frac{\partial}{\partial t}, \tilde{X}\right], \frac{\partial \tilde{f}}{\partial t}\right)$.

Now, if $\tilde{\alpha}$ is a time-dependent 1 -form on $M, \tilde{X}$ is a time-dependent vector field and $\left(x_{0}, t_{0}\right) \in M \times \mathbb{R}$ then, using the isomorphism $T_{\left(x_{0}, t_{0}\right)}^{*}(M \times \mathbb{R}) \cong T_{x_{0}}^{*} M \oplus T_{t_{0}}^{*} \mathbb{R}$, it follows that

$$
\left(\tilde{\mathcal{L}}_{\tilde{X}} \tilde{\alpha}\right)_{\left(x_{0}, t_{0}\right)}=\left(\mathcal{L}_{\tilde{X}_{t_{0}}} \tilde{\alpha}_{t_{0}}\right)_{\left(x_{0}\right)}+\tilde{\alpha}_{\left(x_{0}, t_{0}\right)}\left(\frac{\partial \tilde{X}}{\partial t}{ }_{\mid\left(x_{0}, t_{0}\right)}\right) d t_{\mid t_{0}}
$$

where $\tilde{\mathcal{L}}$ (resp. $\mathcal{L})$ is the Lie derivative on $M \times \mathbb{R}($ resp. $M)$.

Moreover, $\tilde{\mathcal{L}}_{\frac{\partial}{\partial t}} \tilde{\alpha}$ is a time-dependent 1 -form on $M$ and if $\tilde{f} \in C^{\infty}(M \times \mathbb{R}, \mathbb{R})$, then $(\tilde{\alpha}, \tilde{f})$ is a time-dependent section of the vector bundle $T^{*} M \times \mathbb{R} \rightarrow M$ and

$$
\frac{\partial(\tilde{\alpha}, \tilde{f})}{\partial t}=\left(\tilde{\mathcal{L}}_{\frac{\partial}{\partial t}} \tilde{\alpha}, \frac{\partial \tilde{f}}{\partial t}\right) .
$$

A long computation, using (2.9), (2.11), (4.24) and (4.25), shows that

$$
\begin{aligned}
& \llbracket(\tilde{\alpha}, \tilde{f}),(\tilde{\beta}, \tilde{g}) \rrbracket_{(\Lambda, E)}^{X_{0}}=\llbracket \Phi^{*}(\tilde{\alpha}+\tilde{f} d t), \Phi^{*}(\tilde{\beta}+\tilde{g} d t) \rrbracket_{(\Lambda, E)}^{X_{0}}=\llbracket \tilde{\alpha}+\tilde{f} d t, \tilde{\beta}+\tilde{g} d t \rrbracket_{\tilde{\Lambda}} \\
& \widetilde{\#}(\Lambda, E)^{X_{0}}(\tilde{\alpha}, \tilde{f})=\widetilde{\#(\Lambda, E)} X_{0}\left(\Phi^{*}(\tilde{\alpha}+\tilde{f} d t)\right)=\#_{\tilde{\Lambda}}(\tilde{\alpha}+\tilde{f} d t),
\end{aligned}
$$

for $\tilde{\alpha}, \tilde{\beta}$ time-dependent 1 -forms on $M$ and $\tilde{f}, \tilde{g} \in C^{\infty}(M \times \mathbb{R}, \mathbb{R})$, where $\tilde{\Lambda}$ is the Poissonization of the Jacobi structure $(\Lambda, E)$ and $\Phi^{*}: T^{*}(M \times \mathbb{R}) \rightarrow \tilde{A}^{*}=A^{*} \times \mathbb{R}$ is the adjoint isomorphism of $\Phi$.

Therefore, $\tilde{A}^{*}=A^{*} \times \mathbb{R}$ can be identified with $T^{*}(M \times \mathbb{R})$ and, under this identification, the Lie algebroid structure $\left(\llbracket, \rrbracket_{(\Lambda, E)}^{X_{0}}, \widetilde{\#_{(\Lambda, E)}} X_{0}\right)$ is just the Lie algebroid structure $\left(\llbracket, \rrbracket_{\tilde{\Lambda}}, \#_{\tilde{\Lambda}}\right)$ on $T^{*}(M \times \mathbb{R})$.

Consequently, using Proposition 4.10, we deduce that, for this particular case, the pair $\left(\tilde{A}, \tilde{A}^{*}\right)$ is a Lie bialgebroid, when we consider on $\tilde{A}$ and $\tilde{A}^{*}$ the Lie algebroid structures $\left([,]^{-\phi_{0}}, \bar{\pi}^{\phi_{0}}\right)$ and $\left(\llbracket, \mathbb{\rrbracket}_{(\Lambda, E)}^{X_{0}}, \widetilde{\mathbb{\#}_{(\Lambda, E)}} X_{0}\right)$, respectively.

In this section, we generalize the above result for an arbitrary generalized Lie bialgebroid. In fact, we prove

Theorem 4.11 Let $\left(\left(A, \phi_{0}\right),\left(A^{*}, X_{0}\right)\right)$ be a generalized Lie bialgebroid and $(\Lambda, E)$ the induced Jacobi structure over $M$. Consider on $\tilde{A}$ (resp. $\left.\tilde{A}^{*}\right)$ the Lie algebroid structure $\left(\llbracket, \rrbracket^{-\phi_{0}}, \bar{\rho}^{\phi_{0}}\right)$ (resp. $\left.\left(\llbracket, \rrbracket_{*}^{X X_{0}},{\widehat{\rho_{*}}}^{X_{0}}\right)\right)$. Then: 
i) The pair $\left(\tilde{A}, \tilde{A}^{*}\right)$ is a Lie bialgebroid over $M \times \mathbb{R}$.

ii) If $\tilde{\Lambda}$ is the induced Poisson structure on $M \times \mathbb{R}$ then $\tilde{\Lambda}$ is the Poissonization of the Jacobi structure $(\Lambda, E)$.

Proof: i) Using (4.18) and (4.19), we obtain that

$$
\begin{aligned}
&{\widehat{d_{*}}}^{X_{0}} \llbracket \tilde{X}, \tilde{Y} \rrbracket^{-\phi_{0}=} e^{-t}\left(d_{* X_{0}} \llbracket \tilde{X}, \tilde{Y} \rrbracket+d_{*} X_{0}\left(\phi_{0}(\tilde{X}) \frac{\partial \tilde{Y}}{\partial t}\right)-d_{* X_{0}}\left(\phi_{0}(\tilde{Y}) \frac{\partial \tilde{X}}{\partial t}\right)\right. \\
&\left.+X_{0} \wedge \frac{\partial}{\partial t} \llbracket \tilde{X}, \tilde{Y} \rrbracket+X_{0} \wedge \frac{\partial}{\partial t}\left(\phi_{0}(\tilde{X}) \frac{\partial \tilde{Y}}{\partial t}\right)-X_{0} \wedge \frac{\partial}{\partial t}\left(\phi_{0}(\tilde{Y}) \frac{\partial \tilde{X}}{\partial t}\right)\right) .
\end{aligned}
$$

Moreover, applying (3.4), (4.13), (4.14) and (4.15), it follows that

$$
\begin{aligned}
&{\widehat{d_{*}}}^{X_{0}} \llbracket \tilde{X}, \tilde{Y} \rrbracket^{-\phi_{0}}=e^{-t}\left(d_{* X_{0}} \llbracket \tilde{X}, \tilde{Y} \rrbracket+X_{0} \wedge \llbracket \frac{\partial \tilde{X}}{\partial t}, \tilde{Y} \rrbracket+X_{0} \wedge \llbracket \tilde{X}, \frac{\partial \tilde{Y}}{\partial t} \rrbracket+\phi_{0}(\tilde{X}) \frac{\partial}{\partial t}\left(d_{* X_{0}} \tilde{Y}\right)\right. \\
&-\phi_{0}(\tilde{Y}) \frac{\partial}{\partial t}\left(d_{*} X_{0} \tilde{X}\right)-\phi_{0}(\tilde{X}) X_{0} \wedge \frac{\partial \tilde{Y}}{\partial t}+\phi_{0}(\tilde{Y}) X_{0} \wedge \frac{\partial \tilde{X}}{\partial t} \\
&+\phi_{0}(\tilde{X}) X_{0} \wedge \frac{\partial^{2} \tilde{Y}}{\partial t^{2}}-\phi_{0}(\tilde{Y}) X_{0} \wedge \frac{\partial^{2} \tilde{X}}{\partial t^{2}}+\frac{\partial}{\partial t}\left(\phi_{0}(\tilde{X})\right) X_{0} \wedge \frac{\partial \tilde{Y}}{\partial t} \\
&\left.-\frac{\partial}{\partial t}\left(\phi_{0}(\tilde{Y})\right) X_{0} \wedge \frac{\partial \tilde{X}}{\partial t}+d_{* X_{0}}\left(\phi_{0}(\tilde{X})\right) \wedge \frac{\partial \tilde{Y}}{\partial t}-d_{* X_{0}}\left(\phi_{0}(\tilde{Y})\right) \wedge \frac{\partial \tilde{X}}{\partial t}\right) .
\end{aligned}
$$

On the other hand, from (4.3), (4.13), (4.18) and (4.22), we have

$$
\begin{aligned}
\llbracket \tilde{X},{\widehat{d_{*}}}^{X_{0}} \tilde{Y} \rrbracket^{-\phi_{0}}=e^{-t} & \left(\llbracket \tilde{X}, d_{* X_{0}} \tilde{Y} \rrbracket_{\phi_{0}}+\llbracket \tilde{X}, X_{0} \wedge \frac{\partial \tilde{Y}}{\partial t} \rrbracket_{\phi_{0}}+\phi_{0}(\tilde{X}) \frac{\partial}{\partial t}\left(d_{* X_{0}} \tilde{Y}\right)\right. \\
& \left.+\phi_{0}(\tilde{X}) X_{0} \wedge \frac{\partial^{2} \tilde{Y}}{\partial t^{2}}-\frac{\partial \tilde{X}}{\partial t} \wedge i_{\phi_{0}}\left(d_{* X_{0}} \tilde{Y}\right)+\phi_{0}\left(\frac{\partial \tilde{Y}}{\partial t}\right) \frac{\partial \tilde{X}}{\partial t} \wedge X_{0}\right) .
\end{aligned}
$$

Therefore, using (3.20) and (3.22) and the fact that $\frac{\partial}{\partial t}\left(\phi_{0}(\tilde{Z})\right)=\phi_{0}\left(\frac{\partial \tilde{Z}}{\partial t}\right)$, for $\tilde{Z} \in \Gamma(\tilde{A})$,

$$
\begin{aligned}
& \llbracket \tilde{X},{\widehat{d_{*}}}^{X_{0}} \tilde{Y} \rrbracket^{-\phi_{0}}=e^{-t}\left(\llbracket \tilde{X}, d_{* X_{0}} \tilde{Y} \rrbracket_{\phi_{0}}+\llbracket \tilde{X}, X_{0} \rrbracket \wedge \frac{\partial \tilde{Y}}{\partial t}+X_{0} \wedge \llbracket \tilde{X}, \frac{\partial \tilde{Y}}{\partial t} \rrbracket-\phi_{0}(\tilde{X}) X_{0} \wedge \frac{\partial \tilde{Y}}{\partial t}\right. \\
&+\phi_{0}(\tilde{X}) \frac{\partial}{\partial t}\left(d_{*} X_{0} \tilde{Y}\right)+\phi_{0}(\tilde{X}) X_{0} \wedge \frac{\partial^{2} \tilde{Y}}{\partial t^{2}}-\frac{\partial \tilde{X}}{\partial t} \wedge i_{\phi_{0}}\left(d_{* X_{0}} \tilde{Y}\right) \\
&\left.+\frac{\partial}{\partial t}\left(\phi_{0}(\tilde{Y})\right) \frac{\partial \tilde{X}}{\partial t} \wedge X_{0}\right) .
\end{aligned}
$$

Finally, from (4.1) and (4.4), we deduce that

$$
{\widehat{d_{*}}}^{X_{0}} \llbracket \tilde{X}, \tilde{Y} \rrbracket^{-\phi_{0}}=\llbracket \tilde{X},{\widehat{d_{*}}}^{X_{0}} \tilde{Y} \rrbracket^{-\phi_{0}}-\llbracket \tilde{Y},{\widehat{d_{*}}}^{X_{0}} \tilde{X} \rrbracket^{-\phi_{0}} .
$$

ii) Using (4.3), (4.17), (4.20) and Theorem 4.6, we obtain that the induced Poisson structure $\tilde{\Lambda}$ on $M \times \mathbb{R}$ is given by

$$
\tilde{\Lambda}(\delta \tilde{f}, \delta \tilde{g})={\widehat{d_{*}}}^{X_{0}} \tilde{f} \cdot \bar{d}^{\phi_{0}} \tilde{g}=e^{-t}\left(d \tilde{g} \cdot d_{*} \tilde{f}+\frac{\partial \tilde{f}}{\partial t} \rho\left(X_{0}\right)(\tilde{g})-\frac{\partial \tilde{g}}{\partial t} \rho\left(X_{0}\right)(\tilde{f})\right),
$$

for $\tilde{f}, \tilde{g} \in C^{\infty}(M \times \mathbb{R}, \mathbb{R})$.

On the other hand, using (4.11) and (4.12), we prove that

$$
e^{-t}\left(\Lambda+\frac{\partial}{\partial t} \wedge E\right)(\tilde{f}, \tilde{g})=e^{-t}\left(d \tilde{g} \cdot d_{*} \tilde{f}+\frac{\partial \tilde{f}}{\partial t} \rho\left(X_{0}\right)(\tilde{g})-\frac{\partial \tilde{g}}{\partial t} \rho\left(X_{0}\right)(\tilde{f})\right),
$$

for $\tilde{f}, \tilde{g} \in C^{\infty}(M \times \mathbb{R}, \mathbb{R})$. Therefore, $\tilde{\Lambda}$ is the Poissonization of $(\Lambda, E)$.

$Q E D$

Now, we discuss a converse of Theorem 4.11. 
Theorem 4.12 Let $(A, \llbracket, \rrbracket, \rho)$ be a Lie algebroid and $\phi_{0} \in \Gamma\left(A^{*}\right)$ a 1-cocycle. Suppose that $\left(\llbracket, \rrbracket_{*}, \rho_{*}\right)$ is a Lie algebroid structure on $A^{*}$ and that $X_{0} \in \Gamma(A)$ is a 1-cocycle. Consider on $\tilde{A}=A \times \mathbb{R}$ (resp. $\left.\tilde{A}^{*}=A^{*} \times \mathbb{R}\right)$ the Lie algebroid structure $\left(\llbracket, \rrbracket^{-\phi_{0}}, \bar{\rho}^{\phi_{0}}\right)$ (resp. $\left.\left(\llbracket, \rrbracket_{*}^{X_{0}},{\widehat{\rho_{*}}}^{X_{0}}\right)\right)$. If $\left(\tilde{A}, \tilde{A}^{*}\right)$ is a Lie bialgebroid then the pair $\left(\left(A, \phi_{0}\right),\left(A^{*}, X_{0}\right)\right)$ is a generalized Lie bialgebroid.

Proof: Let $\{,\}^{\sim}$ be the induced Poisson bracket on $M \times \mathbb{R}$. Then, from (4.17), (4.20) and Theorem 4.6, it follows that

$$
\{\tilde{f}, \tilde{g}\}^{\sim}=e^{-t}\left(d \tilde{g} \cdot d_{*} \tilde{f}+\frac{\partial \tilde{f}}{\partial t} \rho\left(X_{0}\right)(\tilde{g})+\frac{\partial \tilde{g}}{\partial t} \rho_{*}\left(\phi_{0}\right)(\tilde{f})+\frac{\partial \tilde{g}}{\partial t} \frac{\partial \tilde{f}}{\partial t} \phi_{0}\left(X_{0}\right)\right),
$$

for $\tilde{f}, \tilde{g} \in C^{\infty}(M \times \mathbb{R}, \mathbb{R})$. Since $\{,\}^{\sim}$ is skew-symmetric, we have that $\{t, t\}^{\sim}=0$ which implies that $\phi_{0}\left(X_{0}\right)=0$. As a consequence,

$$
\{\tilde{f}, \tilde{g}\}^{\sim}=e^{-t}\left(d \tilde{g} \cdot d_{*} \tilde{f}+\frac{\partial \tilde{f}}{\partial t} \rho\left(X_{0}\right)(\tilde{g})+\frac{\partial \tilde{g}}{\partial t} \rho_{*}\left(\phi_{0}\right)(\tilde{f})\right) .
$$

In particular, if $f \in C^{\infty}(M, \mathbb{R})$ then, using that $\{f, t\}^{\sim}=-\{t, f\}^{\sim}$, we conclude that $\rho\left(X_{0}\right)=-\rho_{*}\left(\phi_{0}\right)$.

Now, if $X, Y \in \Gamma(A)$, from (4.18), (4.19) and (4.22), we obtain that

$$
\begin{aligned}
\widehat{d}_{*}^{X_{0}} \llbracket X, Y \rrbracket^{-\phi_{0}} & =e^{-t} d_{* X_{0}} \llbracket X, Y \rrbracket, \\
\llbracket X, \widehat{d}_{*}^{X_{0}} Y \rrbracket^{-\phi_{0}}-\llbracket Y,{\widehat{d_{*}}}^{X_{0}} X \rrbracket^{-\phi_{0}}= & \bar{\rho}^{\phi_{0}}(X)\left(e^{-t}\right) d_{* X_{0}} Y+e^{-t} \llbracket X, d_{* X_{0}} Y \rrbracket^{-\phi_{0}} \\
& -\bar{\rho}^{\phi_{0}}(Y)\left(e^{-t}\right) d_{* X_{0}} X-e^{-t} \llbracket Y, d_{* X_{0}} X \rrbracket^{-\phi_{0}} \\
= & e^{-t}\left(\llbracket X, d_{* X_{0}} Y \rrbracket_{\phi_{0}}-\llbracket Y, d_{* X_{0}} X \rrbracket_{\phi_{0}}\right) .
\end{aligned}
$$

Thus, since ${\widehat{d_{*}}}^{X_{0}} \llbracket X, Y \rrbracket^{-\phi_{0}}=\llbracket X,{\widehat{d_{*}}}^{X_{0}} Y \rrbracket^{-\phi_{0}}-\llbracket Y, \widehat{d}_{*}^{X_{0}} X \rrbracket^{-\phi_{0}}$, we deduce (4.1).

Finally, if $X \in \Gamma(A)$ then, using the computations in the proof of Theorem 4.11 and the fact that

$$
{\widehat{d_{*}}}^{X_{0}} \llbracket X, e^{t} Y \rrbracket^{-\phi_{0}}=\llbracket X,{\widehat{d_{*}}}^{X_{0}}\left(e^{t} Y\right) \rrbracket^{-\phi_{0}}-\llbracket e^{t} Y,{\widehat{d_{*}}}^{X_{0}} X \rrbracket^{-\phi_{0}},
$$

for all $Y \in \Gamma(A)$, we prove that $\left(\llbracket X_{0}, X \rrbracket+\left(\mathcal{L}_{* X_{0}}\right)_{\phi_{0}} X\right) \wedge Y=0$. But this implies that

$$
\llbracket X_{0}, X \rrbracket+\left(\mathcal{L}_{* X_{0}}\right)_{\phi_{0}} X=0 .
$$

$Q E D$

In [23] it was proved that if the pair $\left(A, A^{*}\right)$ is a Lie bialgebroid then the pair $\left(A^{*}, A\right)$ is also a Lie bialgebroid. Using this fact, Propositions 4.7 and 4.10 and Theorems 4.11 and 4.12, we conclude that a similar result holds for generalized Lie bialgebroids.

Theorem 4.13 If $\left(\left(A, \phi_{0}\right),\left(A^{*}, X_{0}\right)\right)$ is a generalized Lie bialgebroid, so is $\left(\left(A^{*}, X_{0}\right)\right.$, $\left.\left(A, \phi_{0}\right)\right)$. 


\section{$5 \quad$ Triangular generalized Lie bialgebroids}

Let $(A, \llbracket, \rrbracket, \rho)$ be a Lie algebroid over $M$ and $\phi_{0} \in \Gamma\left(A^{*}\right)$ a 1-cocycle. Moreover, let $P \in \Gamma\left(\wedge^{2} A\right)$ be a bisection satisfying

$$
\llbracket P, P \rrbracket_{\phi_{0}}=0 \text {. }
$$

We shall discuss what happens on the dual bundle $A^{*} \rightarrow M$. Remark 3.3 i) and Remark 3.4 i) suggest us to introduce the bracket $\llbracket, \rrbracket_{* P}$ on $\Gamma\left(A^{*}\right)$ defined by

$$
\begin{aligned}
\llbracket \phi, \psi \rrbracket_{* P} & =\left(\mathcal{L}_{\phi_{0}}\right)_{\#_{P}(\phi)} \psi-\left(\mathcal{L}_{\phi_{0}}\right)_{\#_{P}(\psi)} \phi-d_{\phi_{0}}(P(\phi, \psi)) \\
& =i_{\#_{P}(\phi)} d_{\phi_{0}} \psi-i_{\#_{P}(\psi)} d_{\phi_{0}} \phi+d_{\phi_{0}}(P(\phi, \psi)),
\end{aligned}
$$

for $\phi, \psi \in \Gamma\left(A^{*}\right)$.

Theorem 5.1 Let $(A, \llbracket, \rrbracket, \rho)$ a Lie algebroid over $M, \phi_{0} \in \Gamma\left(A^{*}\right)$ a 1-cocycle and $P \in$ $\Gamma\left(\wedge^{2} A\right)$ a bisection of $A \rightarrow M$ satisfying $\llbracket P, P \rrbracket_{\phi_{0}}=0$. Then:

i) The dual bundle $A^{*} \rightarrow M$ together with the bracket defined in (5.1) and the bundle map $\rho_{* P}=\rho \circ \#_{P}: A^{*} \rightarrow T M$ is a Lie algebroid.

ii) $X_{0}=-\#_{P}\left(\phi_{0}\right) \in \Gamma(A)$ is a 1-cocycle.

iii) The pair $\left(\left(A, \phi_{0}\right),\left(A^{*}, X_{0}\right)\right)$ is a generalized Lie bialgebroid.

Proof: If we consider the Lie algebroid structure $\left(\llbracket, \rrbracket^{-\phi_{0}}, \bar{\rho}^{\phi_{0}}\right)$ on $\tilde{A}=A \times \mathbb{R} \rightarrow M \times \mathbb{R}$ and the bisection $\tilde{P}=e^{-t} P \in \Gamma\left(\wedge^{2} \tilde{A}\right)$ then, from (4.19), 4.20) and Theorem 3.5, it follows that $\llbracket \tilde{P}, \tilde{P} \rrbracket^{-\phi_{0}}=0$. Thus, using the results of Mackenzie and $\mathrm{Xu}$ [23], we deduce that the vector bundle $\tilde{A}^{*} \rightarrow M \times \mathbb{R}$ admits a Lie algebroid structure $\left(\llbracket, \rrbracket^{\sim}, \tilde{\rho}\right)$, where $\tilde{\rho}: \tilde{A}^{*} \rightarrow T(M \times \mathbb{R})$ is the bundle map given by $\tilde{\rho}=\bar{\rho}^{\phi_{0}} \circ \#_{\tilde{P}}$ and $\llbracket, \rrbracket^{\sim}$ is the bracket on $\Gamma\left(\tilde{A}^{*}\right)$ defined by

$$
\llbracket \tilde{\phi}, \tilde{\psi} \rrbracket^{\sim}=i_{\#_{\tilde{P}}(\tilde{\phi})} \bar{d}^{\phi_{0}} \tilde{\psi}-i_{\#_{\tilde{P}}(\tilde{\psi})} \bar{d}^{\phi_{0}} \tilde{\phi}+\bar{d}^{\phi_{0}}(\tilde{P}(\tilde{\phi}, \tilde{\psi}))
$$

for $\tilde{\phi}, \tilde{\psi} \in \Gamma\left(\tilde{A}^{*}\right)$.

Now, we will prove that

$$
\llbracket \tilde{\phi}, \tilde{\psi} \rrbracket_{* P}^{X_{0}}=e^{-t}\left(\llbracket \tilde{\phi}, \tilde{\psi} \rrbracket_{* P}+\tilde{\phi}\left(X_{0}\right)\left(\frac{\partial \tilde{\psi}}{\partial t}-\tilde{\psi}\right)-\tilde{\psi}\left(X_{0}\right)\left(\frac{\partial \tilde{\phi}}{\partial t}-\tilde{\phi}\right)\right)=\llbracket \tilde{\phi}, \tilde{\psi} \rrbracket^{\tilde{y}}
$$

From (3.2), (5.1) and the definition of $X_{0}$, we have that

$$
\begin{aligned}
\llbracket \tilde{\phi}, \tilde{\psi} \rrbracket_{* P}^{X_{0}}= & e^{-t}\left(i_{\#_{P}(\tilde{\phi})} d \tilde{\psi}-i_{\#_{P}(\tilde{\psi})} d \tilde{\phi}+d(P(\tilde{\phi}, \tilde{\psi}))+P(\tilde{\psi}, \tilde{\phi}) \phi_{0}\right. \\
& \left.+\left(i_{\#_{P}(\tilde{\phi})} \phi_{0}\right) \frac{\partial \tilde{\psi}}{\partial t}-\left(i_{\#_{P}(\tilde{\psi})} \phi_{0}\right) \frac{\partial \tilde{\phi}}{\partial t}\right) .
\end{aligned}
$$


Using the fact that

$$
\frac{\partial}{\partial t}(P(\tilde{\phi}, \tilde{\psi}))=P\left(\frac{\partial \tilde{\phi}}{\partial t}, \tilde{\psi}\right)+P\left(\tilde{\phi}, \frac{\partial \tilde{\psi}}{\partial t}\right)=i_{\#_{P}(\tilde{\phi})} \frac{\partial \tilde{\psi}}{\partial t}-i_{\#_{P}(\tilde{\psi})} \frac{\partial \tilde{\phi}}{\partial t},
$$

we obtain that,

$$
\begin{aligned}
\llbracket \tilde{\phi}, \tilde{\psi} \rrbracket_{* P}^{X_{0}}= & e^{-t}\left(i_{\#_{P}(\tilde{\phi})}\left(d \tilde{\psi}+\phi_{0} \wedge \frac{\partial \tilde{\psi}}{\partial t}\right)-i_{\#_{P}(\tilde{\psi})}\left(d \tilde{\phi}+\tilde{\phi}_{0} \wedge \frac{\partial \tilde{\phi}}{\partial t}\right)+d(P(\tilde{\phi}, \tilde{\psi}))\right. \\
& \left.-(P(\tilde{\phi}, \tilde{\psi})) \phi_{0}+\frac{\partial}{\partial t}(P(\tilde{\phi}, \tilde{\psi})) \phi_{0}\right) .
\end{aligned}
$$

Thus, from (4.20) and (4.21), we deduce (5.2).

On the other hand, using (4.16) and (4.19), it follows that

$$
\tilde{\rho}(\tilde{\phi})=e^{-t}\left(\rho_{* P}(\tilde{\phi})+X_{0}(\tilde{\phi}) \frac{\partial}{\partial t}\right)=\widehat{\rho_{* P}} X_{0}(\tilde{\phi}),
$$

for $\tilde{\phi} \in \Gamma\left(\tilde{A}^{*}\right)$. Therefore, from Proposition 4.9, we prove $i$ ) and $\left.i i\right)$.

Now, if we consider on $\tilde{A}$ (resp. $\left.\tilde{A}^{*}\right)$ the Lie algebroid structure $\left(\llbracket, \rrbracket^{-\phi_{0}}, \bar{\rho}^{\phi_{0}}\right)$ (resp. $\left(\llbracket, \rrbracket_{* P}^{X_{0}}\right.$, \left.\left.${\widehat{\rho_{* P}}}^{X_{0}}\right)\right)$ then the pair $\left(\tilde{A}, \tilde{A}^{*}\right)$ is a Lie bialgebroid (see [23]). Consequently, using Theorem 4.12, we conclude that $\left(\left(A, \phi_{0}\right),\left(A^{*}, X_{0}\right)\right)$ is a generalized Lie bialgebroid. $Q E D$

Let $(A, \llbracket, \rrbracket, \rho)$ be a Lie algebroid and $\phi_{0} \in \Gamma\left(A^{*}\right)$ a 1-cocycle. Suppose that $\left(\llbracket, \rrbracket_{*}, \rho_{*}\right)$ is a Lie algebroid structure on $A^{*}$ and that $X_{0} \in \Gamma(A)$ is a 1-cocycle. Moreover, assume that $\left(\left(A, \phi_{0}\right),\left(A^{*}, X_{0}\right)\right)$ is a generalized Lie bialgebroid. Then, the pair $\left(\left(A, \phi_{0}\right),\left(A^{*}, X_{0}\right)\right)$ is said to be a triangular generalized Lie bialgebroid if there exists $P \in \Gamma\left(\wedge^{2} A\right)$ such that $\llbracket P, P \rrbracket_{\phi_{0}}=0$ and

$$
\llbracket, \rrbracket_{*}=\llbracket, \rrbracket_{* P}, \quad \rho_{*}=\rho_{*_{P}}, \quad X_{0}=-\#_{P}\left(\phi_{0}\right) .
$$

Note that a triangular generalized Lie bialgebroid $\left(\left(A, \phi_{0}\right),\left(A^{*}, X_{0}\right)\right)$ such that $\phi_{0}=0$ is just a triangular Lie bialgebroid (see [23]). On the other hand, if $(M, \Lambda, E)$ is a Jacobi manifold then, using Remarks 3.3 and 3.4, we deduce that the pair $\left((T M \times \mathbb{R},(0,1)),\left(T^{*} M \times \mathbb{R}\right.\right.$, $(-E, 0)))$ is a triangular generalized Lie bialgebroid.

\section{Generalized Lie bialgebras}

In this Section, we will study generalized Lie bialgebroids over a point.

Definition 6.1 A generalized Lie bialgebra is a generalized Lie bialgebroid over a point, that is, a pair $\left(\left(\mathfrak{g}, \phi_{0}\right),\left(\mathfrak{g}^{*}, X_{0}\right)\right)$, where $(\mathfrak{g},[]$,$) is a real Lie algebra of finite dimension$ such that the dual space $\mathfrak{g}^{*}$ is also a Lie algebra with Lie bracket $[,]_{*}, X_{0} \in \mathfrak{g}$ and $\phi_{0} \in \mathfrak{g}^{*}$ are 1-cocycles on $\mathfrak{g}^{*}$ and $\mathfrak{g}$, respectively, and

$$
\begin{gathered}
d_{* X_{0}}[X, Y]=\left[X, d_{* X_{0}} Y\right]_{\phi_{0}}-\left[Y, d_{* X_{0}} X\right]_{\phi_{0}}, \\
\phi_{0}\left(X_{0}\right)=0 \\
i_{\phi_{0}}\left(d_{*} X\right)+\left[X_{0}, X\right]=0,
\end{gathered}
$$

for all $X, Y \in \mathfrak{g}, d_{*}$ being the differential on $\left(\mathfrak{g}^{*},[,]_{*}\right)$. 
Remark 6.2 In the particular case when $\phi_{0}=0$ and $X_{0}=0$, we recover the concept of a Lie bialgebra, that is, a dual pair $\left(\mathfrak{g}, \mathfrak{g}^{*}\right)$ of Lie algebras such that

$$
d_{*}[X, Y]=\left[X, d_{*} Y\right]-\left[Y, d_{*} X\right]
$$

for $X, Y \in \mathfrak{g}($ see $[5])$.

Next, we give different methods to obtain generalized Lie bialgebras.

Proposition 6.3 Let $\left(\mathfrak{h},[,]_{\mathfrak{h}}\right)$ be a Lie algebra, $r \in \wedge^{2} \mathfrak{h}$ and $\bar{X}_{0} \in \mathfrak{h}$ such that

$$
[r, r]_{\mathfrak{h}}-2 \bar{X}_{0} \wedge r=0, \quad\left[\bar{X}_{0}, r\right]_{\mathfrak{h}}=0 .
$$

Then, if $\mathfrak{g}=\mathfrak{h} \times \mathbb{R}$, the pair $\left((\mathfrak{g},(0,1)),\left(\mathfrak{g}^{*},\left(-\bar{X}_{0}, 0\right)\right)\right)$ is a generalized Lie bialgebra.

Proof: Consider on $\mathfrak{g}$ the Lie bracket [, ] given by

$$
[(X, \lambda),(Y, \mu)]=\left([X, Y]_{\mathfrak{h}}, 0\right)
$$

for $(X, \lambda),(Y, \mu) \in \mathfrak{g}$. One easily follows that $\phi_{0}=(0,1) \in \mathfrak{h}^{*} \times \mathbb{R} \cong \mathfrak{g}^{*}$ is a 1 -cocycle. On the other hand, the space $\wedge^{2} \mathfrak{g}=\wedge^{2}(\mathfrak{h} \times \mathbb{R})$ can be identified with the product $\wedge^{2} \mathfrak{h} \times \mathfrak{h}$ (see Section 2.2) and, using (2.4), (6.4), (6.5) and Theorem 3.5, we have that $P=\left(r, \bar{X}_{0}\right) \in$ $\wedge^{2} \mathfrak{h} \times \mathfrak{h} \cong \wedge^{2} \mathfrak{g}$ satisfies

$$
[P, P]_{\phi_{0}}=0 .
$$

Therefore, from Theorem 5.1, we deduce that there exists a Lie bracket on $\mathfrak{g}^{*}$ and the pair $\left((\mathfrak{g},(0,1)),\left(\mathfrak{g}^{*},-\#_{P}(0,1)\right)\right.$ is a generalized Lie bialgebra. Moreover, we have that $\#_{P}(0,1)=\left(\bar{X}_{0}, 0\right)$ (see (2.4) and (2.8)). Q

Remark 6.4 $\quad$ i) Note that this method of finding generalized Lie bialgebras is related to find algebraic Jacobi structures.

ii) Using (2.3), (2.4) and (5.1), it follows that the Lie bracket $[,]_{*}$ on $\mathfrak{g}^{*}$ is given by

$$
\begin{aligned}
{[(\alpha, \lambda),(\beta, \mu)]_{*}=} & \left(\operatorname{coad}_{\#_{r}(\alpha)} \beta-\operatorname{coad}_{\#_{r}(\beta)} \alpha-i_{\bar{X}_{0}}(\alpha \wedge \beta)\right. \\
& \left.-\mu \operatorname{coad}_{\bar{X}_{0}} \alpha+\lambda \operatorname{coad}_{\bar{X}_{0}} \beta,-r(\alpha, \beta)\right),
\end{aligned}
$$

for $(\alpha, \lambda),(\beta, \mu) \in \mathfrak{g}^{*}$, where coad $: \mathfrak{h} \times \mathfrak{h}^{*} \rightarrow \mathfrak{h}^{*}$ is the coadjoint representation of $\mathfrak{h}$ over $\mathfrak{h}^{*}$ defined by $\left(\operatorname{coad}_{X} \alpha\right)(Y)=-\alpha[X, Y]$, for $X, Y \in \mathfrak{h}$ and $\alpha \in \mathfrak{h}^{*}$.

Corollary 6.5 Let $\left(\mathfrak{h},[,]_{\mathfrak{h}}\right)$ be a Lie algebra and $\mathcal{Z}(\mathfrak{h})$ the center of $\mathfrak{h}$. If $r \in \wedge^{2} \mathfrak{h}, \bar{X}_{0} \in$ $\mathcal{Z}(\mathfrak{h})$ and

$$
[r, r]_{\mathfrak{h}}-2 \bar{X}_{0} \wedge r=0,
$$

then the pair $\left((\mathfrak{h}, 0),\left(\mathfrak{h}^{*},-\bar{X}_{0}\right)\right)$ is a generalized Lie bialgebra. 
Proof: Using Proposition 6.3, we have that $\left((\mathfrak{g}=\mathfrak{h} \times \mathbb{R},(0,1)),\left(\mathfrak{g}^{*}=\mathfrak{h}^{*} \times \mathbb{R},\left(-\bar{X}_{0}, 0\right)\right)\right)$ is a generalized Lie bialgebra. Furthermore, from (6.6) and since $\bar{X}_{0} \in \mathcal{Z}(\mathfrak{h})$, we deduce that the Lie bracket on $\mathfrak{g}^{*}$ is given by

$$
[(\alpha, \lambda),(\beta, \mu)]_{*}=\left(\operatorname{coad}_{\#_{r}(\alpha)} \beta-\operatorname{coad}_{\#_{r}(\beta)} \alpha-i_{\bar{X}_{0}}(\alpha \wedge \beta),-r(\alpha, \beta)\right),
$$

for $(\alpha, \lambda),(\beta, \mu) \in \mathfrak{g}^{*}$. Then $\mathfrak{h}$ and $\mathfrak{h}^{*}$ are Lie algebras, where the Lie bracket on $\mathfrak{h}^{*}$ is defined by

$$
[\alpha, \beta]_{\mathfrak{h}^{*}}=\operatorname{coad}_{\#_{r}(\alpha)} \beta-\operatorname{coad}_{\#_{r}(\beta)} \alpha-i_{\bar{X}_{0}}(\alpha \wedge \beta),
$$

for $\alpha, \beta \in \mathfrak{h}^{*}$. Moreover, using (6.5), (6.7), (6.8) and the fact that $\left((\mathfrak{g},(0,1)),\left(\mathfrak{g}^{*},\left(-\bar{X}_{0}, 0\right)\right)\right)$ is a generalized Lie bialgebra, we conclude that $\left((\mathfrak{h}, 0),\left(\mathfrak{h}^{*},-\bar{X}_{0}\right)\right)$ is a generalized Lie bialgebra.

Remark 6.6 From (6.8) and Corollary 6.5, we deduce a well-known result (see [5]): if $\left(\mathfrak{h},[,]_{\mathfrak{h}}\right)$ is a Lie algebra, $r \in \wedge^{2} \mathfrak{h}$ is a solution of the classical Yang-Baxter equation (that is, $\left.[r, r]_{\mathfrak{h}}=0\right)$ and on $\mathfrak{h}^{*}$ we consider the bracket defined by

$$
[\alpha, \beta]_{\mathfrak{h}^{*}}=\operatorname{coad}_{\#_{r}(\alpha)} \beta-\operatorname{coad}_{\#_{r}(\beta)} \alpha, \text { for } \alpha, \beta \in \mathfrak{h}^{*},
$$

then the pair $\left(\mathfrak{h}, \mathfrak{h}^{*}\right)$ is a Lie bialgebra.

Examples 6.7 $\quad$ i) Let $\left(\mathfrak{h},[,]_{\mathfrak{h}}\right)$ be the Lie algebra of the Heisenberg group $H(1,1)$. Then, $\mathfrak{h}=<\left\{e_{1}, e_{2}, e_{3}\right\}>$ and

$$
\left[e_{1}, e_{2}\right]_{\mathfrak{h}}=e_{3}, \quad e_{3} \in \mathcal{Z}(\mathfrak{h}) .
$$

If we take $r=e_{1} \wedge e_{2}$ and $\bar{X}_{0}=-e_{3}$, we have that $[r, r]_{\mathfrak{h}}-2 \bar{X}_{0} \wedge r=0$ and thus, using Corollary 6.5, we conclude that $\left((\mathfrak{h}, 0),\left(\mathfrak{h}^{*}, e_{3}\right)\right)$ is a generalized Lie bialgebra. Note that $r$ and $\bar{X}_{0}$ induce the canonical left-invariant contact structure of $H(1,1)$.

ii) Denote by $\mathfrak{h}=\mathfrak{s u}(2)=\left\{A \in g l(2, \mathbb{C}) / \bar{A}^{T}=-A, \operatorname{trace} A=0\right\}$ the Lie algebra of the special unitary group $S U(2)$ and by $\sigma_{1}, \sigma_{2}$ and $\sigma_{3}$ the Pauli matrices

$$
\sigma_{1}=\left(\begin{array}{ll}
0 & 1 \\
1 & 0
\end{array}\right) \quad \sigma_{2}=\left(\begin{array}{cc}
0 & -i \\
i & 0
\end{array}\right) \quad \sigma_{3}=\left(\begin{array}{cc}
1 & 0 \\
0 & -1
\end{array}\right) .
$$

Then, the matrices $\left\{e_{1}=\frac{i}{2} \sigma_{1}, e_{2}=\frac{i}{2} \sigma_{2}, e_{3}=\frac{i}{2} \sigma_{3}\right\}$ form a basis of $\mathfrak{h}=\mathfrak{s u}(2)$ and if $[,]_{\mathfrak{h}}$ is the Lie bracket on $\mathfrak{h}$, we have that

$$
\left[e_{1}, e_{2}\right]_{\mathfrak{h}}=-e_{3}, \quad\left[e_{1}, e_{3}\right]_{\mathfrak{h}}=e_{2}, \quad\left[e_{2}, e_{3}\right]_{\mathfrak{h}}=-e_{1} .
$$

Since $r=e_{1} \wedge e_{2}$ and $\bar{X}_{0}=e_{3}$ satisfy the equations 6.4$),\left((\mathfrak{s u}(2) \times \mathbb{R},(0,1)),\left(\mathfrak{s u}(2)^{*} \times\right.\right.$ $\left.\left.\mathbb{R},\left(-\bar{X}_{0}, 0\right)\right)\right)$ is a generalized Lie bialgebra. Note that if $[$,$] is the Lie bracket on$ $\mathfrak{s u}(2) \times \mathbb{R}$ then, from (6.5), it follows that $(\mathfrak{s u}(2) \times \mathbb{R},[]$,$) is just the Lie algebra of the$ unitary group $U(2)$. Moreover, $r$ and $\bar{X}_{0}$ induce a left-invariant contact structure on $S U(2)$. 
iii) Let $G l(2, \mathbb{R})$ be the general linear group and $\mathfrak{h}=g l(2, \mathbb{R})$ its Lie algebra. A basis of $\mathfrak{h}$ is given by the following matrices

$$
e_{1}=\left(\begin{array}{ll}
0 & 1 \\
0 & 0
\end{array}\right) \quad e_{2}=\left(\begin{array}{ll}
0 & 0 \\
1 & 0
\end{array}\right) \quad e_{3}=\left(\begin{array}{cc}
1 & 0 \\
0 & -1
\end{array}\right) \quad e_{4}=\left(\begin{array}{ll}
1 & 0 \\
0 & 1
\end{array}\right)
$$

If $[,]_{\mathfrak{h}}$ is the Lie bracket on $\mathfrak{h}$, we have that

$$
\left[e_{1}, e_{2}\right]_{\mathfrak{h}}=e_{3}, \quad\left[e_{1}, e_{3}\right]_{\mathfrak{h}}=-2 e_{1}, \quad\left[e_{2}, e_{3}\right]_{\mathfrak{h}}=2 e_{2}, \quad e_{4} \in \mathcal{Z}(\mathfrak{h})
$$

Therefore, if $r=e_{1} \wedge e_{3}+\left(e_{1}-\frac{1}{2} e_{3}\right) \wedge e_{4}$ and $\bar{X}_{0}=-e_{4}$, we deduce that

$$
[r, r]_{\mathfrak{h}}-2 \bar{X}_{0} \wedge r=0
$$

Consequently, $\left((\mathfrak{h}, 0),\left(\mathfrak{h}^{*},-\bar{X}_{0}\right)\right)$ is a generalized Lie bialgebra (see Corollary 6.5).

Acknowledgments. Research partially supported by DGICYT grant PB97-1487 (Spain). D. Iglesias wishes to thank Spanish Ministerio de Educación y Cultura for a FPU grant.

\section{References}

[1] K.H. Bhaskara, K. Viswanath: Poisson algebras and Poisson manifolds, Research Notes in Mathematics, 174, Pitman, London, 1988.

[2] A. Coste, P. Dazord, A. Weinstein: Groupoïdes symplectiques, Pub. Dép. Math. Lyon, 2/A (1987), 1-62.

[3] T.J. Courant: Dirac manifolds, Trans. A.M.S. 319 (1990), 631-661.

[4] P. Dazord, A. Lichnerowicz, Ch.M. Marle: Structure locale des variétés de Jacobi, J. Math. pures et appl., 70 (1991), 101-152.

[5] V.G. Drinfeld: Hamiltonian Lie groups, Lie bialgebras and the geometric meaning of the classical Yang-Baxter equation, Sov. Math. Dokl. 27 (1983), 68-71.

[6] V.G. Drinfeld: Quantum groups, Proc. Internat. Congress Math., Berkeley, (1986), vol.1, 789-820.

[7] B. Fuchssteiner: The Lie algebra structure of degenerate Hamiltonian and bi-Hamiltonian systems, Progr. Theoret. Phys. 68 (1982), 1082-1104.

[8] J. Grabowski, P. Urbánski: Tangent lifts of Poisson and related structures J. Phys. A: Math. Gen. 28 (1995), 6743-6777.

[9] F. Guédira, A. Lichnerowicz: Géométrie des algébres de Lie locales de Kirillov, J. Math. pures et appl., 63 (1984), 407-484.

[10] R. Ibáñez, M. de León, J.C. Marrero and D. Martín de Diego: Co-isotropic and LegendreLagrangian submanifolds and conformal Jacobi morphisms, J. Phys. A: Math. Gen. 30 (1997), 5427-5444. 
[11] D. Iglesias and J.C. Marrero: Some linear Jacobi structures on vector bundles, Preprint (2000), arXiv: math.DG/0007138, To appear in C.R. Acad. Sci. Paris, 330 Sér. I (2000).

[12] D. Iglesias and J.C. Marrero: Generalized Lie bialgebras and Jacobi structures on Lie groups, in preparation.

[13] Y. Kerbrat, Z. Souici-Benhammadi: Variétés de Jacobi et groupoïdes de contact, C.R. Acad. Sci. Paris, 317 Sér. I (1993), 81-86.

[14] A. Kirillov: Local Lie algebras, Russian Math. Surveys, 31 (1976), 55-75.

[15] Y. Kosmann-Schwarzbach: Exact Gerstenhaber algebras and Lie bialgebroids, Acta Appl. Math., 41 (1995), 153-165.

[16] M. de León, B. López, J.C. Marrero and E. Padrón: Lichnerowicz-Jacobi cohomology and homology of Jacobi manifolds: modular class and duality, Preprint (1999), arXiv: math.DG/9910079.

[17] M. de León, J.C. Marrero, E. Padrón: On the geometric quantization of Jacobi manifolds, J. Math. Phys., 38, (12), (1997), 6185-6213.

[18] P. Libermann, Ch. M. Marle: Symplectic Geometry and Analytical Mechanics, Kluwer, Dordrecht, 1987.

[19] A. Lichnerowicz: Les variétés de Poisson et leurs algébres de Lie associées, J. Differential Geometry, 12 (1977), 253-300.

[20] A. Lichnerowicz: Les variétés de Jacobi et leurs algébres de Lie associées, J. Math. pures et appl., 57 (1978), 453-488.

[21] J.-H. Lu, A. Weinstein: Poisson Lie groups, dressing transformations and Bruhat decompositions, J. Differential Geometry 31 (1990), 501-526.

[22] K. Mackenzie: Lie groupoids and Lie algebroids in differential geometry, Cambridge University Press, 1987.

[23] K. Mackenzie, P. Xu: Lie bialgebroids and Poisson groupoids, Duke Math. J. 73 (1994), 415-452.

[24] J.C. Marrero, J. Monterde, E. Padrón: Jacobi-Nijenhuis manifolds and compatible Jacobi structures, C.R. Acad. Sci. Paris, 329 Sér. I (1999), 797-802.

[25] J. Pradines: Théorie de Lie pour les groupoïdes différentiables. Calcul différentiel dans la catégorie des groupoïdes infinitésimaux, C.R. Acad. Sci. Paris, 264 Sér. A (1967), 245-248.

[26] G. Sánchez de Alvarez: Geometric methods of Classical Mechanics applied to Control Theory, Ph.D. Thesis, University of California at Berkeley (1986).

[27] I. Vaisman: Remarkable operators and commutation formulas on locally conformal Kähler manifolds, Compositio Math., 40 (1980), 287-299.

[28] I. Vaisman: Lectures on the Geometry of Poisson Manifolds, Progress in Math. 118, Birkhäuser, Basel, 1994.

[29] I. Vaisman: The BV-algebra of a Jacobi manifold, Preprint (1999), arXiv: math.DG/ 9904112.

[30] A. Weinstein: The local structure of Poisson manifolds, J. Differential Geometry, 18 (1983), 523-557. Errata et Addenda 22 (1985), 255. 